\title{
Removal of masking effect for damage detection of structures
}

\author{
William Soo Lon Wah ${ }^{1,2 *}$, John S Owen ${ }^{1}$, Yung-Tsang Chen ${ }^{2}$, Ahmed Elamin ${ }^{2}$ and Gethin Wyn Roberts ${ }^{3}$ \\ ${ }^{1}$ Faculty of Engineering, The University of Nottingham \\ ${ }^{2}$ Department of Civil Engineering, The University of Nottingham, Ningbo \\ ${ }^{3}$ University of the Faroe Islands
}

\begin{abstract}
Damage detection of civil engineering structures relies heavily on the use of outlier analysis/novelty detection analysis. Generally, data captured from a structure in its normal environmental condition are used to create a model and compute control limits to represent the normal range of variations of damage sensitive features of the structure. However, the training database used usually includes outlier measurements, which may introduce masking effect. These outlier measurements can affect the mean and standard deviation/covariance matrix of the training database, and hence, affect the model and the control limits. As a result, small damage may not be detected. Therefore, this paper proposes an approach of selecting a 'clean' training database for the construction of the baseline of the undamaged structure so as to detect damage at an earlier stage. The approach makes use of Principal Component Analysis and Median Absolute Deviation to identify outlier measurements. This approach can be applied before the application of damage detection methods to allow damage to be detected at an earlier stage. The proposed approach is applied to a numerical beam model and the Z24 Bridge, in Switzerland. The results obtained demonstrate that damage can be detected at an earlier stage using the approach proposed in this paper. The proposed method also allows the determination of the model (e.g. linear or nonlinear) to be used for damage detection.
\end{abstract}

Keywords: Damage detection, outlier analysis, novelty detection analysis, masking effect, environmental and operational conditions, regression analysis, Principal Component Analysis, Median Absolute Deviation, Gaussian Mixture Model

\section{Introduction}

The development of damage detection methods in the past decade is focused on separating/eliminating the effects of the changing environmental and operational condi- ${ }_{25}$ 5 tions from the effects of damage affecting civil engineering structures. This is because the damage sensitive features (e.g. natural frequency) analysed are also affected by the changing environments the structures face, hence leading to false alerts if not considered[1, 2]. A wide range of damage detection methods have been proposed by researchers using different approaches (e.g. regression analysis 3, 4, 5, 6, 7, 8, 9, multivariate statistical tools [10, 11, 12, 13, 14] and a combination of both [15, 16, 17]) to solve these environmental and operational issues.

Although different approaches are adopted, most of them have the same concept of first creating a baseline/model of the undamaged structure using features captured under a range of environmental conditions. Using the baseline/model created, new measurements can be compared ${ }_{40}$ represents how much the new observation deviates from the ideal state of the structure. Then, through the use of an outlier analysis or a novelty detection analysis, the deviation can be classified as a normal measurement or an outlier measurement. An outlier measurement was defined by Hawkins 18] as 'an observation which deviates so much from the other observations as to arouse suspicions that it was generated by a different mechanism'. Thus, for damage detection, this outlier measurement can be attributed to damage of structural components since the features were generated from a new state of the structure.

In both outlier analysis and novelty detection analysis, control limits are created using a training database to represent the normal condition of the structure. By definition, the difference between these two analysis techniques lies in the training database. For the novelty detection analysis, the training database consists of measurements free of outliers, while for the outlier analysis, outliers can be present in the training database 19. For damage detection, since outliers are usually considered to represent damage events, the training database which is composed of undamaged measurements, is usually assumed to be free of outliers. However, even though data are from the undamaged state of the structure, outliers not in the form of damage are gen-
Email address: William.soo@nottingham.edu.cn (William Soo Lon $\mathrm{Wah}^{1,2 *}$ ) 
45 erally present. Fuentes 20 mentioned that outliers from the undamaged structure can be in two forms. The first is when observations manifest themselves as extreme values, which is generally due to high noise level coming from the ${ }_{105}$ data collection process or due to data corruption. This

50 type of outlier data is usually in number but with very large values which may cause a small increase in the variance and control limits as well as moving the mean of the database towards them [4, 20]. The second type is when the ${ }_{110}$ measurements are created through different mechanisms;

55 one example is features gathered from temperature conditions below and above zero degrees. For example, some bridge structures [5, 21, 22, 23 change behaviour when the temperature condition drops below freezing point. This 115 change in behaviour is usually attributed to the increase

60 in stiffness of the structure due to the asphalt layer on the bridge or to the stiffening of the supports [5, 21, 22, 23]. It is important to identify and take into account these outliers in the database to avoid creating a defective model.120 However, as mentioned by Dervilis et al. 4], generally the 65 outlier measurements are not known a priori, and hence, the inclusive approach which consists of including outliers in the training database is usually adopted.

Outlier measurements present in the training database ${ }_{125}$ may cause a masking effect where real outliers (due to 70 damage) are hidden during future analysis, or undamaged measurements are flagged as damaged when they are not 4. The masking effect generally occurs because most control limits in either novelty detection analysis or outlier ${ }_{130}$ analysis are computed using the mean and standard devi75 ation/covariance matrix of the training database. Hence, if the outliers increase the standard deviation or move the mean towards them, the sensitivity of the method to small damage will be reduced. Therefore, it is important to135 identify and remove these outlier measurements to have

so a 'clean' database for the computation of the control limits. The term 'clean' is adopted here to represent free of outliers.

Outlier measurements can also affect the baseline/modelko constructed to obtain the deviation index. For example, one popular technique adopted to obtain the deviation index is the Mahalanobis Square Distance 24. It relies heavily on the mean and covariance of the features of the undamaged data set. Thus, if outlier measurements are present, the model will be affected. This also usually oc90 curs in other damage detection methods, such as the regression analysis method. The model created through the regression analysis will tend towards the outlier measurements, creating a defective model. Therefore, these outliers need to be identified and removed at the beginning

${ }_{95}$ before creating any baseline/model, and adopt a more ro-150 bust model (usually nonlinear) to tackle the second type of outliers (measurements coming from different environmental mechanisms).

Damage detection methods presented in the literature

can give the presence of damage under changing environ-155 mental and operational conditions. However, no assess- ment has been given to the lowest level of damage that can be detected. This is because structures can be subjected to a wide range of damage scenarios and types, thus, it is difficult to define the severity of damage for each scenario. If the lowest damage severity that can be identified through the damage detection methods already makes the structure unsafe or the scenario can be easily seen through visual inspection (severe cracking or loss of pier), then the methods become redundant. Hence, the factor that should be considered and improved is to make these methods more sensitive to alert damage so that damage can be detected at an earlier stage. This is related to how sensitive the methods are to changes in damage sensitive features and how clean the undamaged database is to represent the undamaged state of the structure. However, having a clean database makes the methods also more prone to alerting undamaged cases as damage, because features with high noise levels will lie outside the control limits.

Therefore, an approach is proposed in this paper to create a clean database to detect damage at an earlier stage whilst not compromising the performance by alerting healthy condition. This approach consists of a data cleaning step where outlier measurements in the training data set are identified and removed before applying damage detection methods. It also allows the determination of the model (linear or nonlinear) to be adopted for the baseline. The approach uses Principal Component Analysis (PCA) and Median Absolute Deviation (MAD) to identify the outliers. This approach can be implemented with existing damage detection methods to improve their sensitivity to detect small damage. To test the proposed approach, two case studies are analysed in this paper; the first is a numerical beam structure model and the second is the Z24 Bridge, in Switzerland, which was subjected to complicated environmental and operational conditions.

The rests of the paper starts with an introduction on the different types of measurements that can be obtained from the undamaged structure. The method to detect outlier measurements with the different mathematical tools used are then introduced. The application of the method to a beam structure model and the Z24 Bridge is then presented. A conclusion then closes the paper.

\section{Introduction on normal and abnormal observa- tions for creating the baseline}

As mentioned previously, measurements from the undamaged state of a structure consist of features captured under a normal range of environmental and operational conditions, and some unwanted outlier measurements. The normal range of conditions may include the daily and seasonal change in ambient temperature conditions, the daily flow of traffic, and the normal wind speed acting on the structure. An outlier observation, in other terms, an abnormal observation not due to damage, may be attributed to several factors. Excessive traffic due to severe traffic congestion or extremely high wind speed due to a cyclone 
may represent events leading to outliers. For example,195 the natural frequencies of the Tamar Bridge, in UK, were found not to be affected by low wind speed, but at high wind speed $(>25 \mathrm{mph})$, the frequencies were affected 25 . These cases are outliers since they rarely occur and hence they need to be identified to be taken into account while 200 constructing the baseline of the undamaged structure.

Another common example of outliers come from noise and processing errors. Farrar et al. 26 mentioned that variabilities in modal testing procedures and data reduction can cause changes in the identified vibration prop-205 erties of a structure. These effects, if high, will affect the measurements greatly leading to outliers. Therefore, these effects also need to be detected before constructing the baseline and computing the control limits of the features of the undamaged structure.

Fig. 11 gives a graphical representation of the different types of measurements that can be obtained from the undamaged condition of a structure. The plot represents one damage sensitive feature versus another feature (e.g. first natural frequency versus second natural frequency).215 It should be noted that the data has not been generated by any model or distribution. The plot represents data gathered continuously, since generally structures are permanently and continuously monitored (i.e. there is no large gap in the environmental and operational conditions that 220 was not monitored).

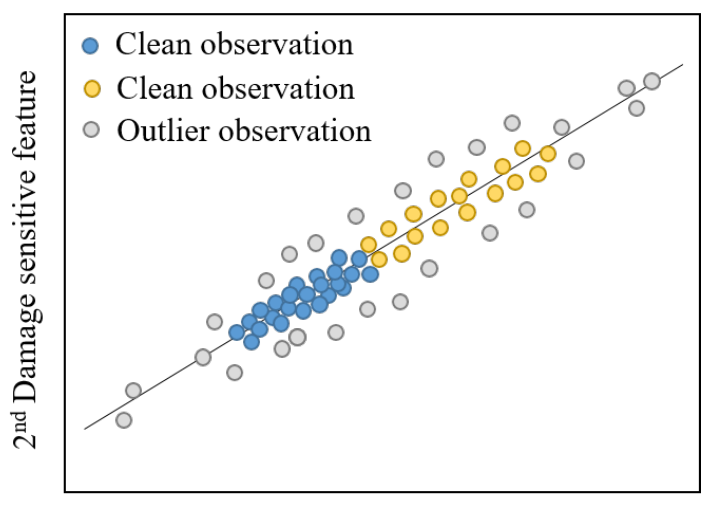

$1^{\text {st }}$ Damage sensitive feature

Figure 1: Types of observations from the undamaged structure.235 *Blue and yellow dots represent the clean observations generated under two different mechanisms and grey dots represent the outliers.

The blue and yellow observations in the plot are the clean observations. Both types of measurements follow the linear model given by the black line. The observations $\mathrm{do}_{240}$ not lie exactly along the linear model due to the presence of noise, variability in modal testing procedures and data reduction, and some minor environmental and operational conditions modifying the features. The blue data set is more compact that the yellow data set. This is because the two data sets represent observations generated by two different mechanisms. In the context of this paper, the different ways the changing environmental and operational conditions (e.g. different temperature range or different wind speed range) affect the features are referred to as the different mechanisms generating the features. A real-life example of two different mechanisms that gives a similar plot is when natural frequencies of a bridge structure are gathered below and above zero degrees [5, 21, 22, 23]. Below freezing point, different structural components contribute to the stiffness of the structure, while above zero degrees, these components contribute less. As a result, the vibration properties are affected differently, and the relationship between temperature and frequencies are different. The effects of different mechanisms affecting the features should be taken into account during the construction of the baseline so as to avoid creating a defective model and giving false alerts.

Another type of measurement is the one given by the grey observations. These observations are due to high noise level, large errors occurring during the extraction process of the features, and some minor environmental and operational effects. The clean data set are surrounded by these unwanted observations. If these observations are included in the training database, the sensitivity of damage detection methods to detect small damage may be reduced. This is because, the standard deviation of the database will be larger and hence, a larger range of normal conditions represented by control limits will be created. Therefore, these grey observations need to be identified and omitted from the baseline. However, it should be noted that if these observations are omitted, a few damage alerts may be raised for the undamaged measurements. A compromise should be made between detecting small damage and not alerting undamaged cases with high noise level, as damage. It should be noted that, flagging outliers due to high noise level are generally not continuous. Hence, a threshold can be established to indicate the amount of continuous ob230 servations outside the control limits to raise the damage alert.

\section{Methodology for identifying outlier measurements}

This section describes the proposed approach for identifying and removing outlier measurements to create the baseline of the undamaged structure. PCA, MAD and Gaussian Mixture Model (GMM) are used in this paper, thus, an introduction to those mathematical tools is first given. The approach proposed in this paper is then described in details.

\subsection{Principal Component Analysis}

PCA is a multivariate statistical tool used to reduce the dimensions of a data set while still retaining most of the information. It is a generative latent variable model where the data being analysed is seen as being generated by a set of latent, unobserved variables 20 . The data here is the damage sensitive features, and the latent variables could represent some mathematical abstractions or actually have physical meaning 20. For example, the latent 
variables may represent the changing environmental and values of damage sensitive features. It may also represent damage of structural components affecting the features.

PCA creates new non-correlated variables called 'principal components' to represent the factors/latent variables principal components are obtained through a rotational transformation of the original data set as shown in Fig. 2. These components highlight the directions of maximum variances in the data set. Mathematically, these principal the variables in the original data set 27 .

$$
Y_{1, j}=L_{1,1} S_{1, j}+L_{1,2} S_{2, j}+\ldots+L_{1, b} S_{b, j}=\sum_{i=1}^{b} L_{1, i} S_{i, j}
$$

where,

$Y_{1, j}$ is the value of the first principal component of the $j^{\text {th }}$ observation of a data set composed of $q$ observations,

$S_{i, j}$ is the $i^{t h}$ feature of the $j^{\text {th }}$ observation of a data set composed of $b$ features captured from $q$ observations, and

$L_{1, i}$ is the $i^{\text {th }}$ coefficient used to compute the first principal component out of $b$ principal components (to reduce dimensions of a data set, only the first $m$ principal components are retained where $m<b$ ).

The coefficients in Eq. (1) are used to compute one principal component only. A set of coefficients is required to compute all the principal components. These coeffi- ${ }^{325}$ cients are grouped in a data set and the set is commonly referred to as the loading matrix (matrix $\mathbf{L}$ with dimensions $b \times b$, and dimensions $m \times b$ if only the first $m$ principal components need to be constructed). This loading matrix corresponds to the eigenvectors of the covariance matrix of 330 the original data set (original data set $\mathbf{S}$ with dimensions $b \times q)$. The covariance matrix is a non-singular matrix and is assumed to be positive semi-definite. This means that the eigenvalues are non-negative. It should be noted that the data set $\mathbf{S}$ should be mean centered before the application of PCA.

The principal components of the matrix $\mathbf{S}$ can therefore ${ }_{335}$ be given as

$$
\mathbf{Y}=\mathbf{L S}
$$

where, $\mathbf{Y}$ (dimensions $b \times q$ and dimensions $m \times q$ if only the first $m$ principal components are retained), called the score matrix, combines all the principal components into one single matrix. In the score matrix, the first principal component accounts for most of the variances of the original data set, the second principal component to second most variances and so on. Therefore, in the loading matrix, the eigenvectors are arranged based on a descending order of their eigenvalues (i.e. the first principal component will have the largest eigenvalue while the last principal component will have the smallest eigenvalue).
For a data set of damage sensitive features, temperature is generally considered to be the dominant environmental effect creating the variations in the features 28 . 5, 29, 30, 25, while other environmental conditions, and noise and errors occurring during data processing, have minor effects. For example, Desjardin et al. 31 found out that the variations of natural frequencies under constant environmental conditions had a standard deviation of 0.5 $\%$ from the mean. This change was attributed to errors and noise occurring in the extraction process of the frequencies. When the structure was subjected to varying environmental conditions, the frequencies varied with a standard deviation of $1.1 \%$ and these frequencies were found to vary linearly with the changing temperature conditions.

Therefore, for a data set of natural frequencies captured under a range of environmental conditions, the first principal component will represent the temperature effect (latent variable) which is creating most of the variations in the data set. The other principal components will represent the other minor effects such as noise and minor environmental conditions (e.g. humidity) affecting the frequencies. To demonstrate this, consider a data set composed of three observations with two features (e.g first and second natural frequencies of a structure).

$$
\mathbf{S}=\left[\begin{array}{lll}
S_{1,1} & S_{1,2} & S_{1,3} \\
S_{2,1} & S_{2,2} & S_{2,3}
\end{array}\right]
$$

where, $S_{i, j}$ represents the $i^{\text {th }}$ feature from the $j^{\text {th }}$ observation.

In this data set, temperature is the main effect defining the values of the features. Other minor environmental effects (e.g. humidity) and errors occurring during the extraction process of the features are then combined together as a single effect and are called noise. Therefore, the features can be obtained as

$$
S_{i, j}=\beta \times T+N E
$$

where,

$T$ is the temperature condition of the $j^{t h}$ observation,

$\beta$ is a coefficient representing the rate of change of the feature due to change in temperature, and

$N E$ is the noise term and has a small range of variation. Substituting Eq. (4) into Eq. (3) yields

$$
\mathbf{S}=\left[\begin{array}{lll}
\left(\beta_{1} T_{1}+N E_{1}\right) & \left(\beta_{1} T_{2}+N E_{2}\right) & \left(\beta_{1} T_{3}+N E_{3}\right) \\
\left(\beta_{2} T_{1}+N E_{4}\right) & \left(\beta_{2} T_{2}+N E_{5}\right) & \left(\beta_{2} T_{3}+N E_{6}\right)
\end{array}\right]
$$

Since two different features are monitored here, two coefficients $\left(\beta_{1}\right.$ and $\left.\beta_{2}\right)$ are used. All the noise terms $\left(N E_{1}\right.$, $\left.N E_{2}, \ldots, N E_{6}\right)$ have different values in the data set.

As mentioned previously, the principal components are arranged based on their eigenvalues which represent the amount of variances the components account for in the original data set. Therefore, these principal components 


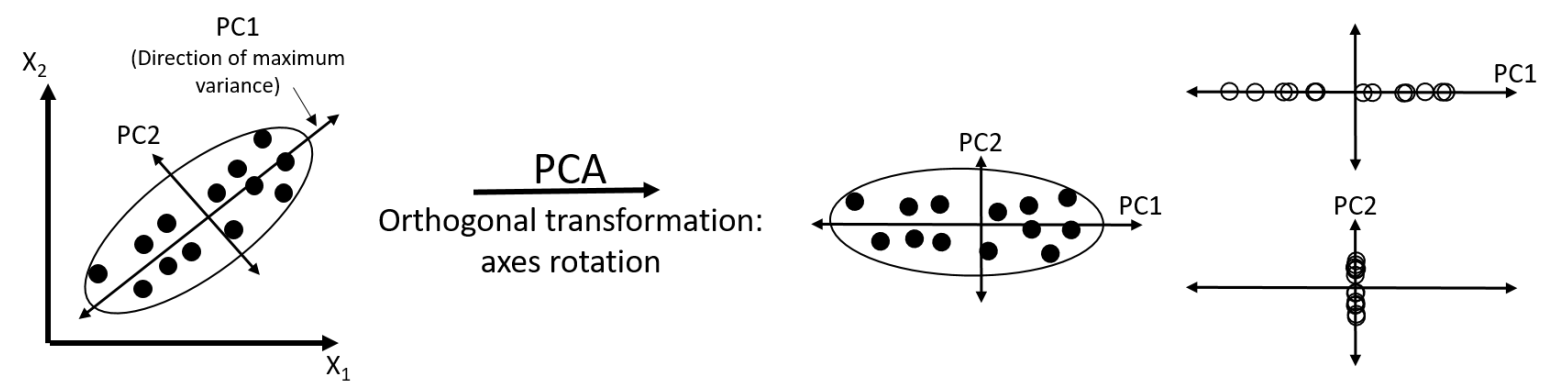

Figure 2: Orthogonal transformation with Principal Component Analysis.

3.2. Median Absolute Deviation

To detect the presence of outliers, the mean plus/minus three standard deviations method is common practice in univariate statistics. This method is based on the char- acteristic that for a normally distributed data set (including both clean and outlier measurements), $99.7 \%$ of the observations will lie within this range and are assumed to be clean. The remaining $0.3 \%$ is then designated as outliers. However, using the mean and standard deviation poses several problems 32. For example, although most features captured from civil engineering structures are usually assumed to follow a normal distribution (or almost normal distribution), the presence of outliers may alter this distribution. Also, the mean and standard deviation are strongly affected by the presence of outliers, which may alter the control limits.

An alternative of using the mean and standard deviation is the median. The median is similar to the mean, a measure of central tendency. However, it has the advantage of being insensitive to the presence of outliers [32]. An indication of insensitivity to outliers is the breakdown point 33 . It is the proportion or amount of (large/small) extreme values that must be introduced into a data set to cause the estimate to yield an arbitrarily bad result. The breakdown point for the median is 0.5 which means that only when more than $50 \%$ of the observations are extreme that the median becomes unreliable. For the mean it is 032 .

MAD, which involves finding the median of absolute deviations from the median given by Eq. (6) 34, can be used to detect the presence of outlying measurements.

$$
\mathrm{MAD}=\text { cons } \times \operatorname{med}\left(\left|A_{j}-\operatorname{med}\left(A_{j}\right)\right|\right)
$$

where,

$A_{j}$ is the data set of the original observation,

med represents median, and

cons is a constant and is linked to the assumption of normality of the data, disregarding the abnormality introduced by the outlier measurements. In this paper, it is assumed that data gathered follows a normal distribution, thus the coefficient is 1.482634 .

Similar to the method of mean plus/minus three standard deviations, the control limits using the MAD method can be computed as follows

$$
\begin{aligned}
& U C L_{\text {med }}=\text { med }+\alpha \mathrm{MAD} \\
& L C L_{\text {med }}=\text { med }-\alpha \mathrm{MAD}
\end{aligned}
$$


Table 1: Values of variables of the data set given in Eq. (5).

\begin{tabular}{lllllllllll}
\hline$N E_{1}$ & $N E_{2}$ & $N E_{3}$ & $N E_{4}$ & $N E_{5}$ & $N E_{6}$ & $\beta_{1}$ & $\beta_{2}$ & $T_{1}$ & $T_{2}$ & $T_{3}$ \\
\hline 0.3 & 0.1 & -0.2 & 0.2 & 0.1 & -0.3 & 0.03 & 0.05 & $1{ }^{\circ} \mathrm{C}$ to $20{ }^{\circ} \mathrm{C}$ & $0{ }^{\circ} \mathrm{C}$ & $-T_{1}$ \\
\hline
\end{tabular}

(a)

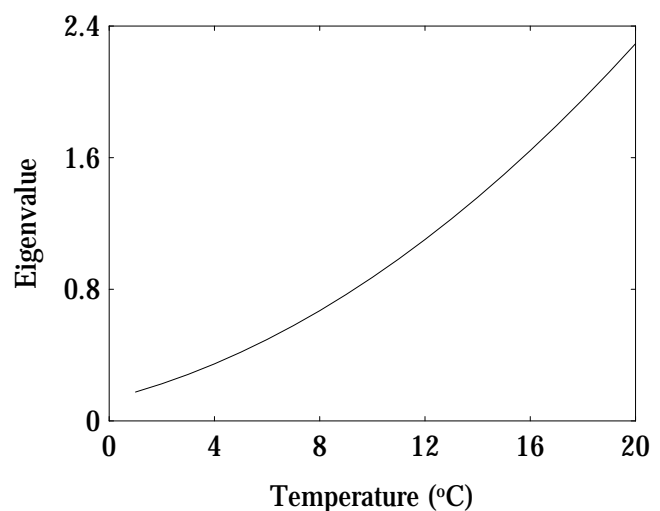

(b)

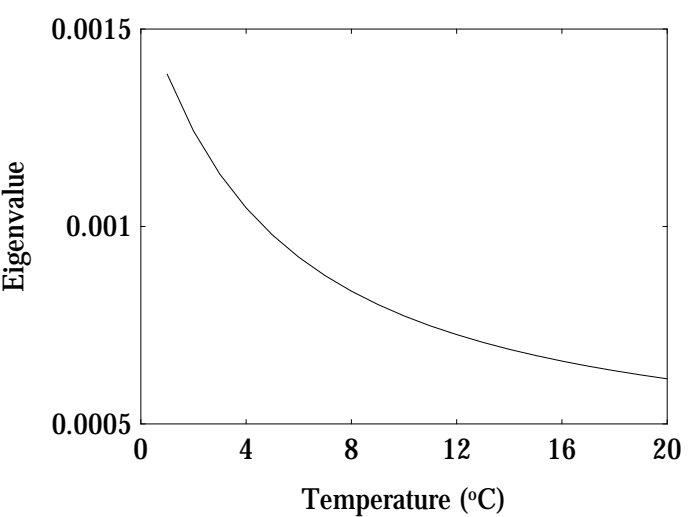

Figure 3: Variations of (a) largest eigenvalue and (b) smallest eigenvalue with temperature for the covariance matrix of the data set given in Eq. (5).

where,

$U C L_{\text {med }}$ is the upper control limit calculated using the MAD method,

$L C L_{\text {med }}$ is the lower control limit calculated using the ${ }_{460}$ MAD method, and

$\alpha$ is a coefficient defining the range of the control limits (usually $2,2.5$ or 3 ) 32 .

To demonstrate the advantage of using MAD over the mean and standard deviation approach, consider a data set (Fig. 4) composed of fifteen observations with values $2,3,4,5,5,5,6,6,6,7,7,8,9,70$ and 100 . Two observations (70 and 100) are outliers which represent around $13 \%$ of the data set. Two outliers are chosen because, if the percentage of outliers was very small and their val- ${ }_{470}$ ues not extreme, the analysis would not be affected by the outliers. The mean of the data set is 16.2 , which is inconsistent with the majority of the observations. The mean plus three standard deviations is calculated as 101.9, and is larger than both extreme observations. The median and the $U C L_{\text {med }}$ ( $\alpha$ value as 3 ) are calculated as 6 and 10.4 , respectively, which are consistent with the majority of the observations. Using the MAD method, the two extreme values are classified as outliers. Thus, if data gathered from civil engineering structures include outliers, MAD is better suited to compute the control limits. In this paper, will be used to identify outliers in the undamaged data set before the application of damage detection methods.

To the authors knowledge, it is the first time that MAD has been used in the context of damage detection of civil engineering structures under changing environmental and operational conditions.

\subsection{Gaussian Mixture Model}

As mentioned previously, damage sensitive features may be generated under different environmental mechanisms. To take this into account, the features can be clustered into different data sets before the applications of damage detection methods, as was adopted by Kerschen and Golinval 35, Yan et al. 36] and Kullaa 37. In this paper GMM is used as the clustering technique. GMM is a probabilistic clustering method which assumes that a data set which is not normally distributed, can be represented by a set of normally distributed components. It is adopted because it uses the mean and covariance of the clusters as basis of clustering. This has the advantage that the relationship between the variables being clustered are taken into account. A brief introduction on GMM is given here.

Consider a multivariate data set $\mathbf{X}$ composed of nonlinear data $\left\{\mathbf{x}_{1}, \ldots, \mathbf{x}_{N}\right\}$ of the likes of natural frequencies captured under bilinear effects of changing temperature conditions from $N$ observations. Nonlinear data are not normally distributed, thus, they cannot be modeled as a single Gaussian standard normal distribution. A mixture of Gaussian components whose distribution can be written as a linear superposition of $K$ Gaussian densities can be assumed to model the data 38 .

$$
p(\mathbf{x})=\sum_{k=1}^{K} \pi_{k} \mathcal{N}\left(\mathbf{x} \mid \boldsymbol{\mu}_{k}, \boldsymbol{\Sigma}_{k}\right)
$$

Each Gaussian component of the mixture given as $\mathcal{N}\left(\mathbf{x} \mid \boldsymbol{\mu}_{k}, \boldsymbol{\Sigma}_{k}\right)$ has its own mean and covariance given as $\boldsymbol{\mu}_{k}$ and $\boldsymbol{\Sigma}_{k}$, respectively. The parameters $\pi_{k}$ in Eq. (8) are called the mixing coefficients, and they ranged between 0 and 1 


\begin{tabular}{|c|c|c|c|}
\hline & & \multicolumn{2}{|c|}{ mean +3 standard deviation } \\
\hline & & & $\bullet$ \\
\hline 1 & 1 & 1 & 1 \\
\hline 40 & 60 & 80 & 100 \\
\hline
\end{tabular}

Figure 4: Plots of the fifteen observations with control limits calculated using MAD, and mean and standard deviation. * Circle represents clean measurement and dot represents outlier measurement.

the likelihood function given in Eq. (9) with respect to the parameters $\left(\boldsymbol{\mu}_{k}, \boldsymbol{\Sigma}_{k}\right.$ and $\left.\pi_{k}\right)$ so as to determine the component each data point $\mathbf{x}_{n}$ belongs to.

$$
\ln p(\mathbf{X} \mid \boldsymbol{\pi}, \boldsymbol{\mu}, \boldsymbol{\Sigma})=\sum_{n=1}^{N} \ln \left\{\sum_{k=1}^{K} \pi_{k} \mathcal{N}\left(\mathbf{x}_{n} \mid \boldsymbol{\mu}_{k}, \boldsymbol{\Sigma}_{k}\right)\right\}
$$

However, these parameters are unknown since it is not known which observation belongs to which component.520 Thus, these unknown parameters can be estimated using the Expectation-Maximization (EM) algorithm. The EM algorithm is an iterative process which is composed of two steps, namely the expectation (E) step and the maximization (M) step. In the E step, the parameters (initial guess ${ }_{525}$ at the beginning) are held fixed and the posterior probability of the component $k$ given the observation $\mathbf{x}_{n}$ is evaluated (called responsibilities $\gamma\left(z_{n k}\right)$ ) as follows

$$
\gamma\left(z_{n k}\right)=\frac{\pi_{k} \mathcal{N}\left(\mathbf{x}_{n} \mid \boldsymbol{\mu}_{k}, \boldsymbol{\Sigma}_{k}\right)}{\sum_{j=1}^{K} \pi_{j} \mathcal{N}\left(\mathbf{x}_{n} \mid \boldsymbol{\mu}_{j}, \boldsymbol{\Sigma}_{j}\right)}
$$

In which $z_{n k}$ is an element of a $K$-dimensional binary random variable $\mathbf{z}$ which has a 1-of- $K$ representation in which a particular element $z_{k}$ is equal to one while all other elements are zero.

Then, in the M step, the parameters are re-estimated using the posterior probability calculated above in the $\mathrm{E}$ step as follows

$$
\begin{gathered}
\boldsymbol{\mu}_{k}^{n e w}=\frac{1}{N_{k}} \sum_{n=1}^{N} \gamma\left(z_{n k}\right) \mathbf{x}_{n} \\
\boldsymbol{\Sigma}_{k}^{n e w}=\frac{1}{N_{k}} \sum_{n=1}^{N} \gamma\left(z_{n k}\right)\left(\mathbf{x}_{n}-\boldsymbol{\mu}_{k}^{n e w}\right)\left(\mathbf{x}_{n}-\boldsymbol{\mu}_{k}^{n e w}\right)^{\mathrm{T}} \\
\pi_{k}^{n e w}=\frac{N_{k}}{N}
\end{gathered}
$$

where,

$$
N_{k}=\sum_{n=1}^{N} \gamma\left(z_{n k}\right)
$$

The log-likelihood given in Eq. (9) can then be evaluated. Convergence of either the parameters or the log likelihood is checked, and if the criteria is not satisfied, ${ }^{555}$ the process will iterate using the up to data values until the criteria is met.

\subsection{Identifying outlier observations approach}

The approach of identifying outliers proposed in this paper is described here. It consists of using PCA to trans5 form the original data set into a new coordinate system to highlight the locations of the clean and outlier observations. As mentioned previously, outliers usually surround the clean observations. These observations cluster separately on the principal component axes. Therefore, this paper proposes to first apply PCA on the features data set before the application of damage detection methods.

After the application of PCA, this paper proposes to rank the observations in an ascending order of their principal component scores to arrange the observations based on their locations relative to one another. Since the outlier observations surround the clean observations, these outliers will cluster together at the two ends of the principal component axes while the clean observations will cluster in the middle portion as demonstrated in Fig. 5 . In the figure, the bold ovals surround the clean observations while the dotted ovals show the outlier observations.

To separate the clean observations from the outliers, this paper proposes to apply MAD analysis on each set of principal component scores. The analysis creates control limits $\left(U C L_{\text {med }}\right.$ and $\left.L C L_{\text {med }}\right)$ that can be used to identify the extreme outlier observations at the two ends of the principal component axes. The advantage of applying MAD analysis on the principal component scores instead of on the damage sensitive features directly is that, each principal component reduces the dimensions of the features data set to a smaller dimension. Worden et al. 24] mentioned that detecting outliers in a multivariate data set is more difficult than the univariate situation because the outliers may hide in the data mass. Therefore, representing the multidimensional features data set by the principal components makes the analysis more sensitive to identifying outliers. Moreover, as mentioned previously, the noise space is represented by the second and lower principal components, and the extreme outliers (grey color in Fig. 11) usually appear in those spaces. Therefore, these lower principal component axes can be used to locate these observations. It is important to clean those spaces since Kullaa 39] mentioned that damage is usually detected in the noise space.

As demonstrated in the previous section, the first principal component highlights the environmental conditions creating the main variance in the features data set. This 


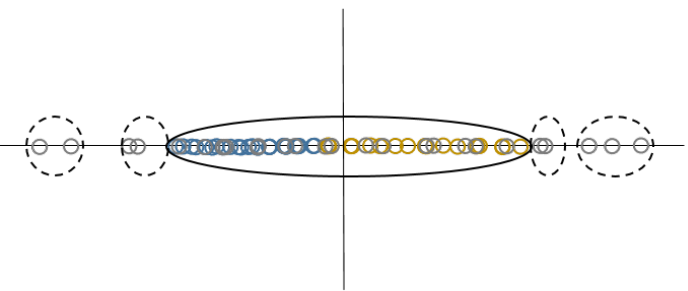

First principal component

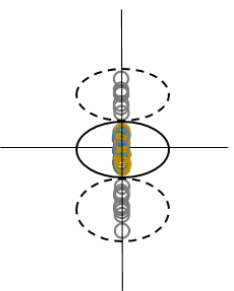

Second principal component

Figure 5: Representation of (a) first principal component and (b) second principal component of the data set given in Fig. 1 *Bold oval shows the clean observations and dotted oval shows the outliers.

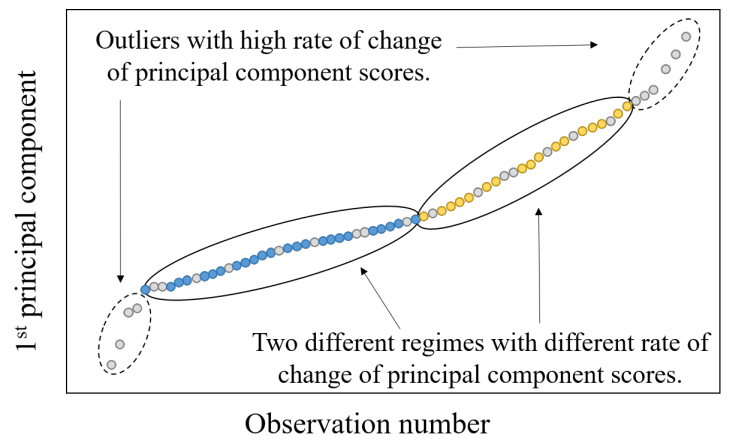

(a) Plot of ranked first principal component versus observation number

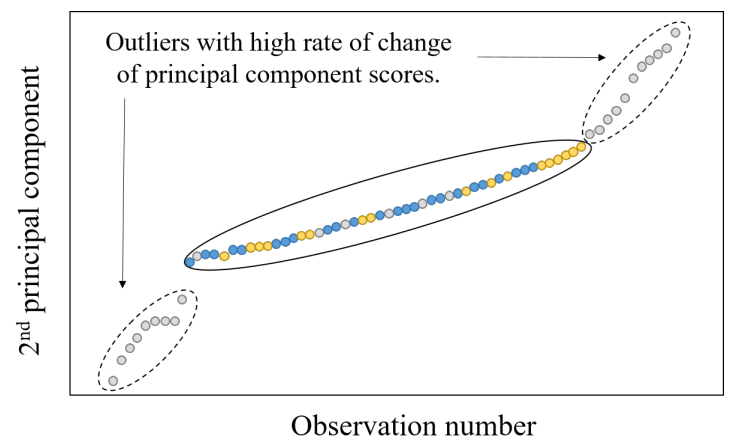

(b) Plot of ranked second principal component versus observation number

Figure 6: Representation (a) first principal component and (b) second principal component arranged against observation number of the data set given in Fig. 1 *Bold oval shows the clean observations and dotted oval shows the outliers.

component can be used to determine whether the data585 set is composed of features generated under the same or different environmental mechanisms. Generally, data captured from civil engineering structures are assumed to follow a normal distribution. This is assuming that only one mechanism is affecting the features. However, if different590 mechanisms are present, then each group of features will have its own normal distribution, and will form clusters in the data set. To determine the number of mechanisms generating the features, this paper proposes to plot the arranged scores of each principal component against obser-595 vation number. On the plot, the observations generated under the same mechanism will cluster together and will have their own pattern as shown in Fig. 6(a). Since the first principal component represents the dominating environmental conditions affecting the features, the different600 environmental mechanisms will appear on the first principal component plot. However, if the different mechanisms also affect the noise space, this will also be reflected on the minor principal component plots. For this case, the noise space is not affected by different mechanisms as the605 second principal component plot (Fig. 6(b)) has only one main cluster of observations. By analysing the principal component plots, an indication on the model (linear or nonlinear) to adopt for damage detection can be obtained. It should be noted that this approach points out the ob-610 servations affected by different environmental effects only.
No indication on the effects themselves is given.

The identified extreme outlier measurements can be removed from the features data set to obtain a clean database to be used for damage detection. The model (linear or nonlinear) to use to create the baseline can be decided and damage detection methods can then be applied on the new database free of outliers to detect damage at an earlier stage.

To take into account nonlinear effects of environmental conditions, two approaches are usually adopted in the literature; either using nonlinear analysis tools 40 or clustering the features into different linear data sets before the application of damage detection methods 35, 36, 37. In this paper, the latter is adopted, and GMM is used as the clustering tool. GMM is applied on the principal component scores highlighting the different mechanisms and the dominating environmental effects affecting the features. The different clusters then represent the different groups of observations affected by different environmental mechanisms. Damage detection methods can then be applied on each group of observations.

To summarise, this paper proposes to apply PCA on the damage sensitive features data set first. It is then proposed to rank the observations based on an ascending order of their principal components scores. To identify whether the features data set is composed of features generated under different mechanisms, this paper proposes to plot the 
arranged scores of each principal component versus obser-635 vation number. Analysing the plots, the different groups of observations (generated by different environmental effects) can be identified. A decision can then be made on the type of model (linear or nonlinear) to adopt for damage detection. In this paper, it is proposed to use GMM to cluster the principal component scores with the environmental conditions affecting the data set so as to group the observations with the same environmental effect, together. It is then proposed to apply MAD analysis on the arranged principal component scores. This will separate the clean observations from the outliers, and these ${ }_{645}$ unwanted measurements can then be discarded from the database. Damage detection methods can then be applied using the data set free of outliers. A flowchart on the procedures to follow to identify outlier measurements is given in Fig. 7 .

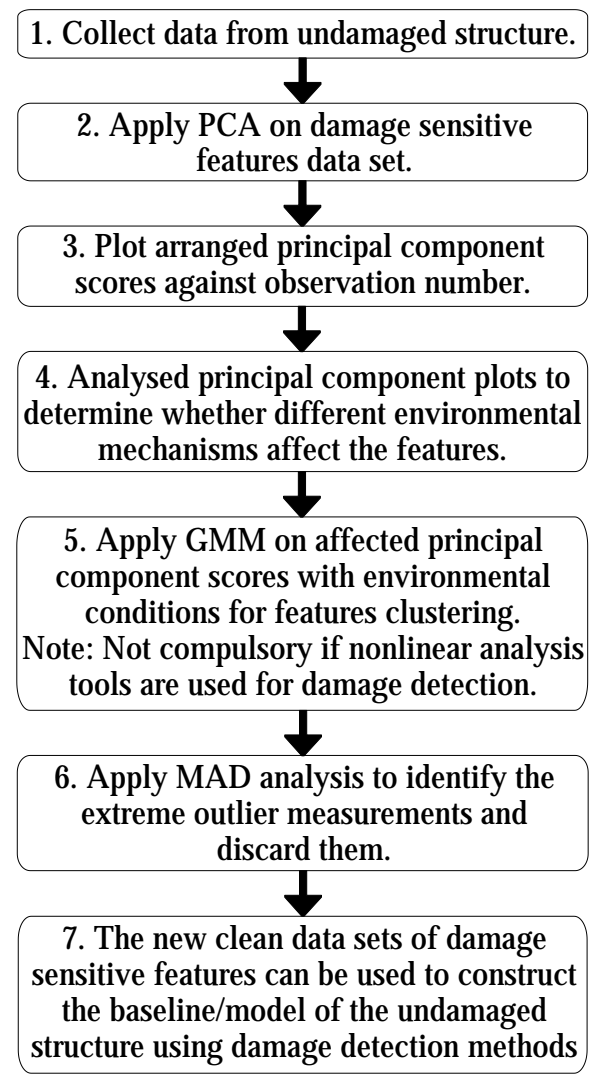

Figure 7: Flow chart of proposed approach of identifying outlier measurements.

\section{Case studies}

To illustrate the proposed approach of identifying outlier measurements to allow detection of damage at an earlier stage, two case studies are examined in this section. The first is a numerical beam model which is subjected ${ }^{60}$ to changing temperature conditions and to varying mass distribution. The second is a real-life bridge structure, the Z24 Bridge, in Switzerland, which was subjected to complicated environmental conditions.

\subsection{Beam structure model}

The beam structure model under consideration is presented in Fig. 8. The structure is $10 \mathrm{~m}$ long and consists of ten beam elements of $1 \mathrm{~m}$ each. The cross-sectional area and second moment of area of the structure are $0.08 \mathrm{~m}^{2}$ and $0.0006 \mathrm{~m}^{4}$, respectively. The Young's modulus of the material is assumed to be temperature dependent. The relationship between the Young's modulus and temperature is assumed to be bilinear (Fig. 9) and is the same as the one proposed by Kullaa[41]. It should be noted that in reality, such a Young's modulus-temperature relationship does not exist. It is adopted here to simulate the common bilinear relationship between natural frequencies and temperature that is usually found in real-life bridge structures. For real-life structures, a combination of effects such as the change in boundary conditions due to thermal effects and the different materials the structures are composed of will define the damage sensitive features. The density of the material is assumed to be $7850 \mathrm{~kg} / \mathrm{m}^{3}$.

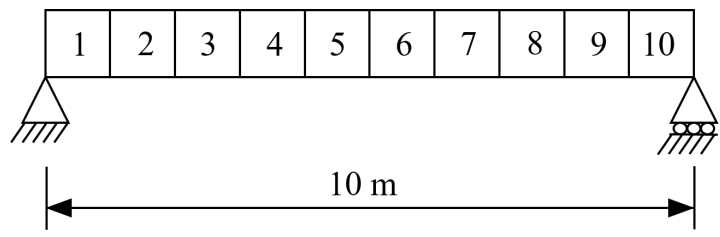

Figure 8: Beam structure model.

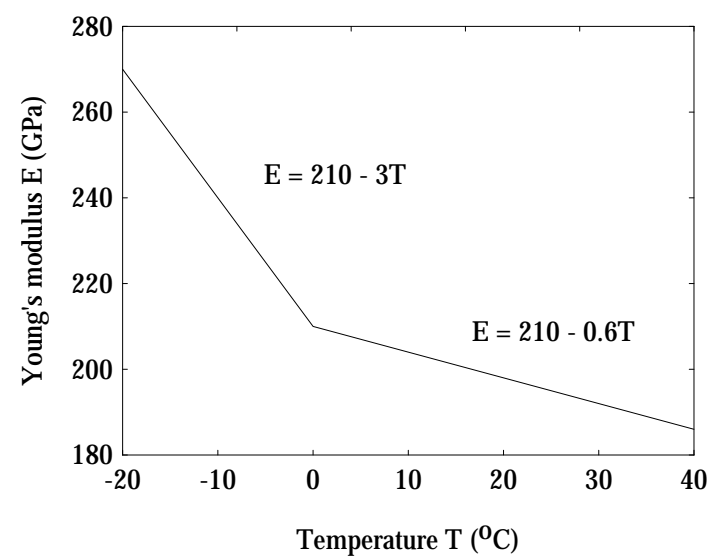

Figure 9: Variations of Young's modulus with temperature for the beam structure model.

The first four natural frequencies of the beam are used as damage sensitive features. The database of the undamaged structure is made of two data sets. The first set includes 1220 observations with 800 observations obtained between temperature conditions of $0{ }^{\circ} \mathrm{C}$ and $40{ }^{\circ} \mathrm{C}$, and 
(a)

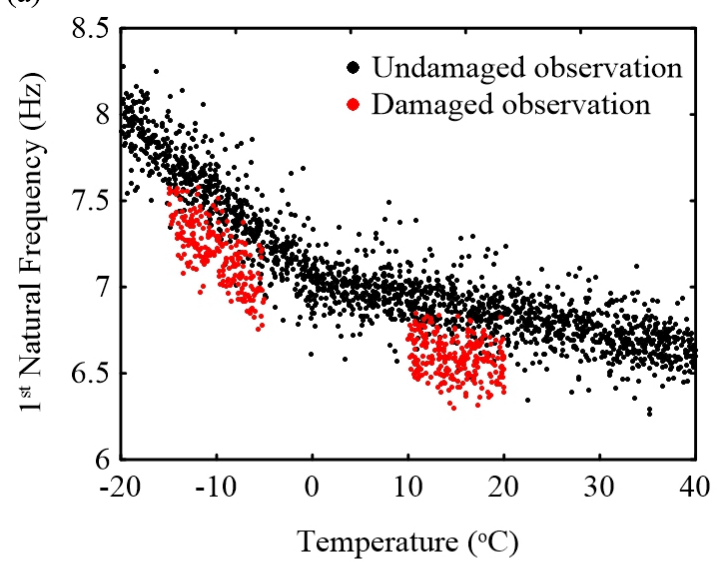

(c)

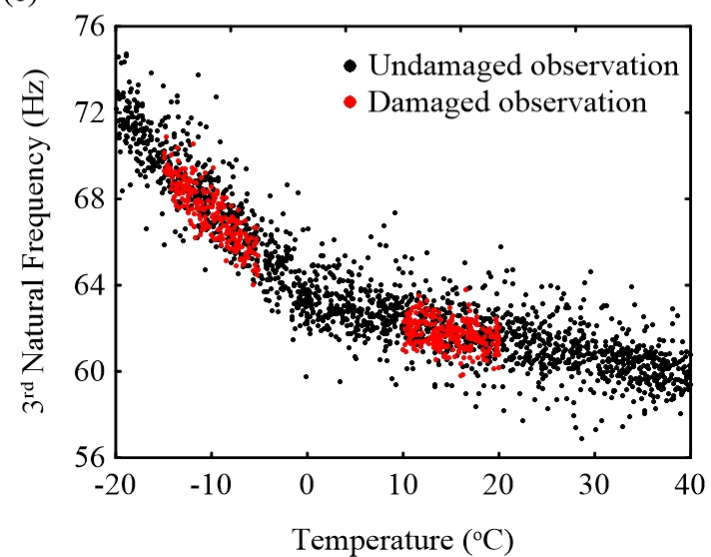

(b)

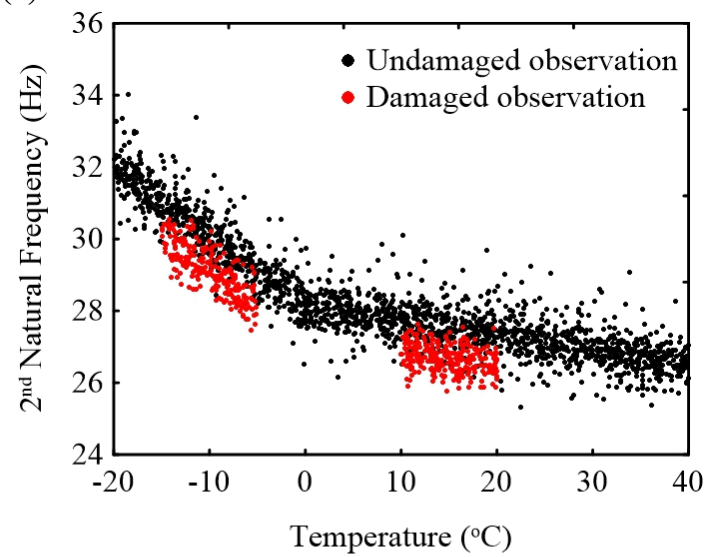

(d)

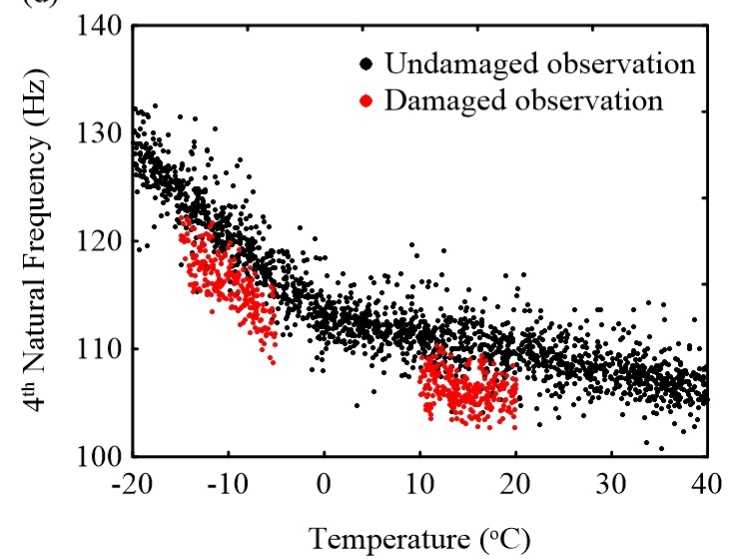

Figure 10: Plot of temperature versus (a) first natural frequency, (b) second natural frequency, (c) thrid natural frequency and (d) fourth natural frequency of undamaged observations and damaged observations (only for temperatures of $10{ }^{\circ} \mathrm{C}$ to $20{ }^{\circ} \mathrm{C}$ and $-5{ }^{\circ} \mathrm{C}$ to $-15^{\circ} \mathrm{C}$ ) of the beam structure model.

the other 420 observations obtained at temperature con-685 ditions between $-20{ }^{\circ} \mathrm{C}$ and $0{ }^{\circ} \mathrm{C}$. For this first set, the density of each element varies by $\pm 10 \%$ from the original density of the material. This variation in mass may represent traffic loading or pedestrian loading on a structure. This creates a variation of the natural frequencies from690 their ideal values. The second data set has 610 observations $\left(400\right.$ from $0{ }^{\circ} \mathrm{C}$ to $40{ }^{\circ} \mathrm{C}$ and 210 from $-20{ }^{\circ} \mathrm{C}$ to $0{ }^{\circ} \mathrm{C}$ ) with a $\pm 25 \%$ variation in density. This second data set is included in the database as a set of outlier measurements. These outlier measurements may move the mean towards695 them and will increase the standard deviation of the undamaged database. It should be noted that although the second data set consists of half of the measurements of the first data set, not all the measurements will act as outliers; some measurements will cluster together with the first data7oo set. This number of observations is chosen because if the number of outliers is insignificant when compared to the clean measurements, it will not affect the mean and standard deviation.

Five damaged cases with increasing severity are applied705 to the structure where damage is simulated as a reduction in elemental stiffness of the $4^{\text {th }}$ element. The reduction severities of the five cases are $25 \%, 30 \%, 35 \%, 40 \%$ and $45 \%$, respectively. These damage severities are chosen because the variations in density $( \pm 10 \%)$ prevent smaller damage severities to be identified. This is because the change in frequencies from the ideal values (no effect from change in density or damage, only effect from temperature) due to the variations in density is larger than those due to damage. Since the purpose of this case study is to demonstrate that damage can be detected at an earlier stage using the proposed approach, these severities are not that important because the case study can demonstrate that damage can be identified at an earlier stage when the proposed approach is applied when compared to the normal situation. Data from each damaged case is assumed to be obtained 200 times with temperature conditions ranging between $0{ }^{\circ} \mathrm{C}$ and $40{ }^{\circ} \mathrm{C}$, and 100 times for conditions between $-20^{\circ} \mathrm{C}$ and $0{ }^{\circ} \mathrm{C}$. Moreover, each element is assumed to have $\mathrm{a} \pm 10 \%$ variation in density.

The plot of temperature conditions versus the four natural frequencies of the undamaged (black) and damaged (red) observations are presented in Fig. 10. Only the damaged observations with temperature conditions between 10 ${ }^{\circ} \mathrm{C}$ and $20{ }^{\circ} \mathrm{C}$, and $-5{ }^{\circ} \mathrm{C}$ and $-15{ }^{\circ} \mathrm{C}$ are included in the 
(a)

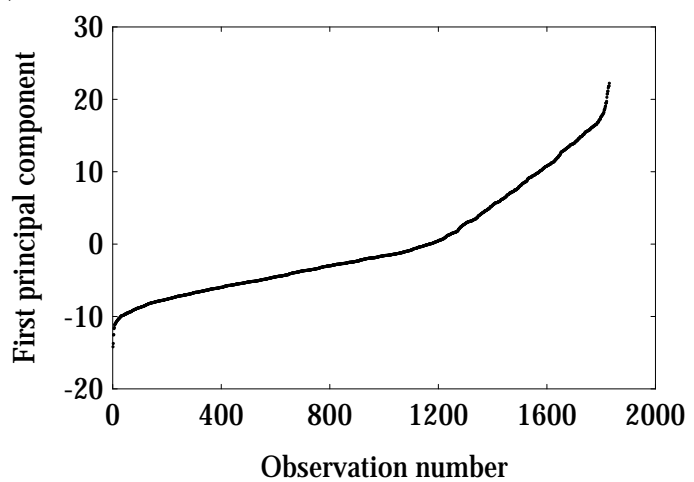

(c)

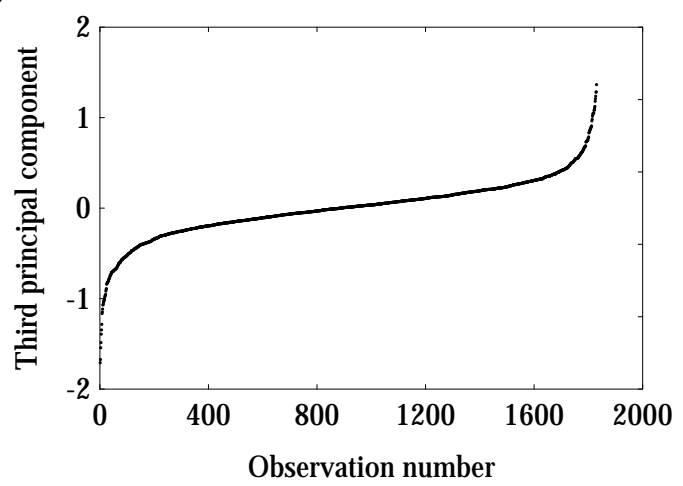

(b)

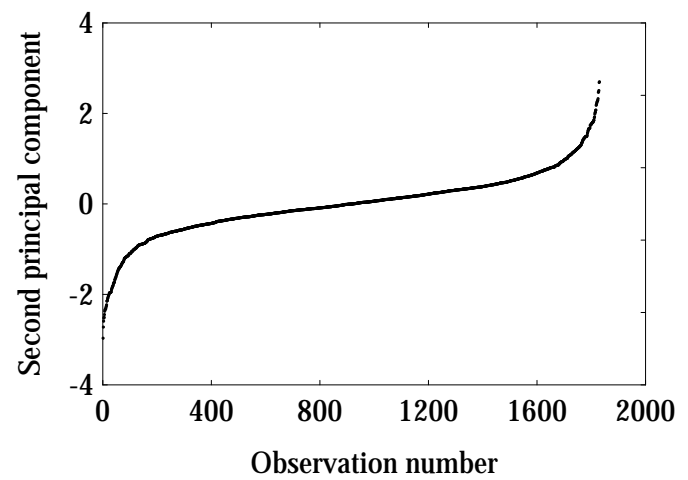

(d)

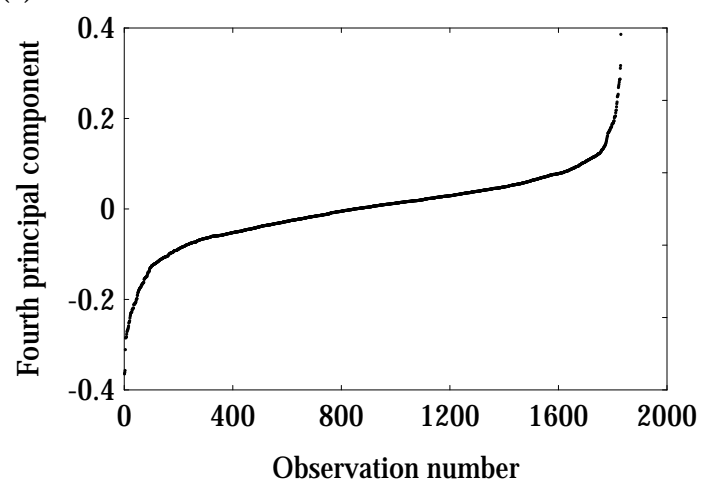

Figure 11: Plot of (a) first principal component, (b) second principal component, (c) third principal component and (d) fourth principal component arranged against observation number for the database of the beam structure model.

figure. This is because, if all the damaged observations were included, the undamaged observations would be hid-735 (In the third natural frequency plot, the damaged and undamaged observations are mixed together and cannot be distinguished from one another. The damaged observations cluster with the undamaged observations because this mode of vibration is relatively 74 the presence of damage. This is because the damage location is near a node of the third mode. For the first, second and fourth natural frequencies, some of the damaged observations cluster together with the undamaged cases. However, most of the damaged observa-745 These outliers are the undamaged measurements that are far away from the majority of the undamaged observations. Therefore, these outliers need to be identified and removed from the database before applying damage detec-750 tion methods.

PCA is applied to all the undamaged observations, and the observations are arranged based on an ascending order of their principal component scores. The plots of principal component scores versus observation number are given in 75

Fig. 11. In the first principal component plot (Fig. 11(a)) two different regions exist which are attributed to two different environmental mechanisms the structure experienced. These two regions represent the observations ob- tained at temperatures below and above $0{ }^{\circ} \mathrm{C}$. Therefore, it is important to separate the observations into two data sets to represent the different conditions, or to use nonlinear data processing techniques while applying damage detection methods. In this paper, the former is adopted and GMM is used to cluster the frequencies into linear regions. GMM is applied on the first principal component plotted against temperature conditions. The clustering is shown in Fig. 12 where the two regions of temperature (blue for temperatures below $0{ }^{\circ} \mathrm{C}$ and red for temperatures above $0{ }^{\circ} \mathrm{C}$ ) can be seen.

In the principal component plots (Fig. 11), the outlier measurements can also be seen at the two ends of each plot. It can be seen that at the two ends, the rate of change of principal component scores is high. This is because outlier measurements usually have extreme values of damage sensitive features with large change between them. Therefore, large change between these observations also occurs with the principal component scores.

To separate the clean measurements from the bad ones, MAD analysis is applied to each set of principal component scores. A coefficient of 2 ( $\alpha$ in Eq. (7)) is used for the control limits in the MAD analysis. The coefficient is taken as 2 to represent the confidence that around $95 \%$ of the observations will lie within the control limits. 2 is chosen instead of the other coefficients so as to make the con- 


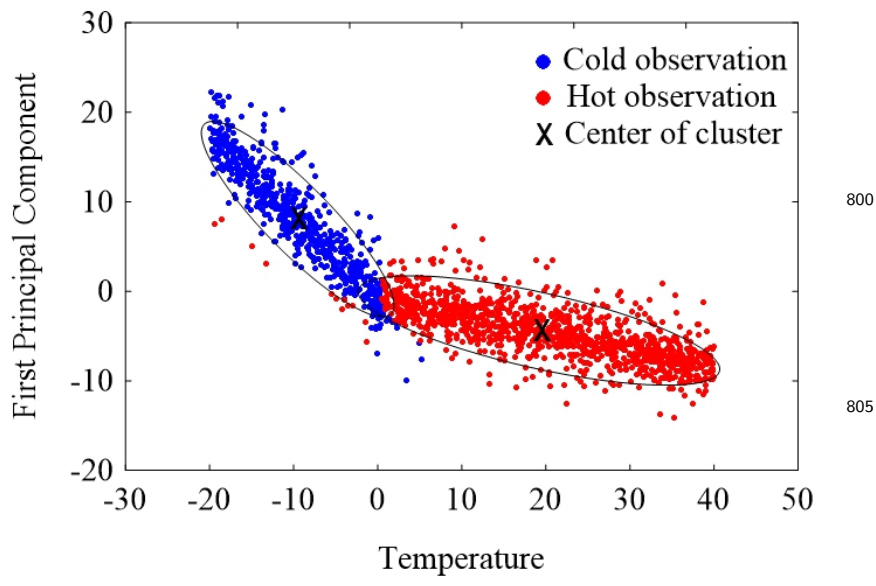

Figure 12: Cluster of first principal component with temperature of the beam structure model using GMM.

trol limits to have a smaller range to identify more outlier ${ }^{810}$ observations. A new database is created after the outlier measurements have been identified and discarded from the original database. From now on, this new database will be referred to as the clean database.

The plots of temperature versus the first four natural ${ }^{815}$ frequencies of the beam sturcture model for the identified outlier undamaged observations and clean undamaged observations are also given in this paper in Fig. 13. The damaged cases (temperature conditions of $10{ }^{\circ} \mathrm{C}$ to $20^{\circ} \mathrm{C}$, and $-5{ }^{\circ} \mathrm{C}$ and $-15^{\circ} \mathrm{C}$ only) are also included in the figure..$^{820}$ It can be seen that most of the outliers given in purple colour surround the clean measurements given in black. Moreover, it can be seen that some of the outlier measurements are mixed together with the clean observations.

775 These measurements cluster together with the clean ob- ${ }^{825}$ servations in some of the plots while in other plots, they are separated from the clean observations and act as extreme value outliers. In the plots it can also be seen that the damaged cases lie in the space of the identified out-

780 lier observations. As mentioned previously, it is important ${ }^{830}$ to clean that space to allow damage to be detected at an earlier stage.

To test whether eliminating the outlier measurements improves the sensitivity of damage detection methods, the inear regression damage detection method is used. This ${ }^{835}$ linear regression method creates a model for natural frequency as a function of temperature conditions. Natural frequencies during future monitoring is predicted using the measured temperature conditions. The residual error between the predicted value and the one obtained from the ${ }^{840}$ structure given in Eq. 15 can be used as a deviation index.

$$
\delta f=f_{p}-f_{o}
$$

where,

$\delta f$ is the residual error between the predicted and the 795 tion index in this paper,

$f_{p}$ is the predicted value of the natural frequency, and $f_{o}$ is the original value of the natural frequency.

An outlier analysis can be performed on this deviation index to classify whether the structure is damaged or not. Since damage detection methods proposed in the literature usually use the mean and standard deviation/covariance matrix to compute the control limits, the mean plus/minus three standard deviations method (Eq. (16) ) is adopted here.

$$
\begin{aligned}
U C L & =\overline{\delta f}+3 \sigma \\
L C L & =\overline{\delta f}-3 \sigma
\end{aligned}
$$

where,

$U C L$ is the upper control limit calculated using the mean and standard deviation,

$L C L$ is the lower control limit calculated using the mean and standard deviation,

$\overline{\delta f}$ is the mean of the residual error $\delta f$, and

$\sigma$ is the standard deviation of the residual error $\delta f$.

The damage detection method is applied using both the clean database and the original database. Damaged observations with temperature conditions below and above 0 ${ }^{\circ} \mathrm{C}$ are analysed separately using their respective database (after clustering was applied to separate the features into two data sets for conditions below and above $0{ }^{\circ} \mathrm{C}$ ). To show samples of the results of the outlier analysis, the five damaged cases with temperature conditions between $0{ }^{\circ} \mathrm{C}$ and $40{ }^{\circ} \mathrm{C}$ are presented in Fig. 14. It should be noted that similar results are obtained for those with temperature conditions below $0{ }^{\circ} \mathrm{C}$.

In Fig. 14, the plots on the left are those computed with the clean database, while those on the right are from the original database. More damaged observations are outside the control limits when using the clean database. The results demonstrate that damage can be detected at an earlier stage when the clean database is adopted. The mean of the residual errors for each case (each damaged severity) is also given in the figure through the bold horizontal line. It can be seen that, for the first natural frequency, the mean starts to move outside the control limit from the third case for the clean database, while for the original database, it is outside only for the last case. Similar results are obtained for the second and fourth frequencies. For the third frequency, since the undamaged and damaged observations are mixed together, the mean is within the control limits. It can also be seen that for all the frequencies, the range of variation represented by the control limits is smaller for the clean database than the original database. This smaller range enables smaller damage scenarios with small deviations from the healthy state of the structure to be identified. Therefore, it is important to remove outlier measurements before applying damage detection methods so that damage can be alerted at an earlier stage. 
(a)

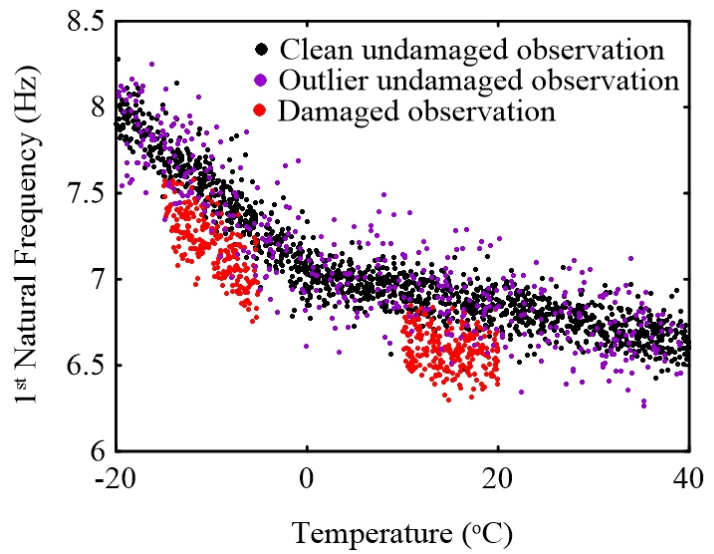

(c)

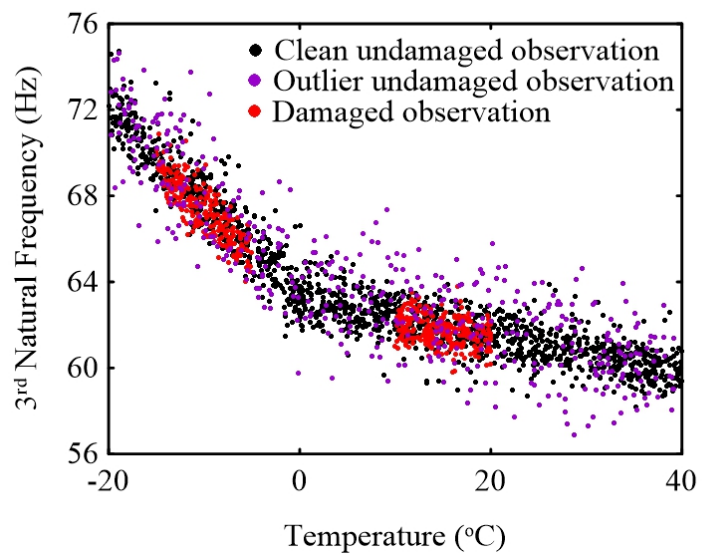

(b)

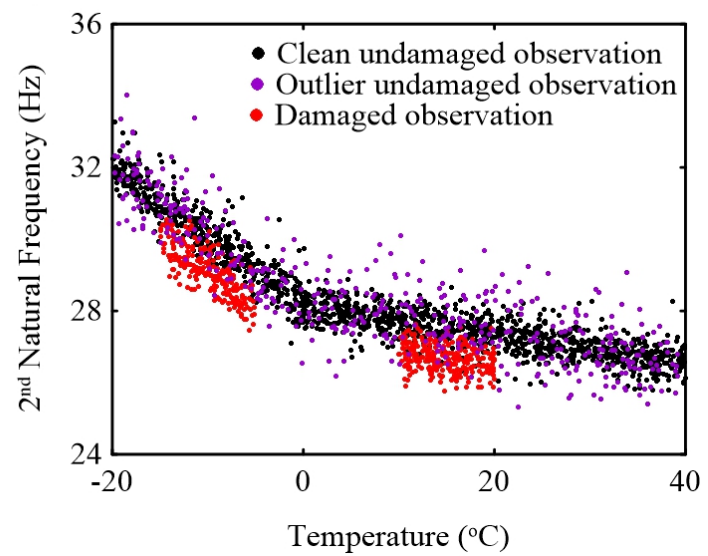

(d)

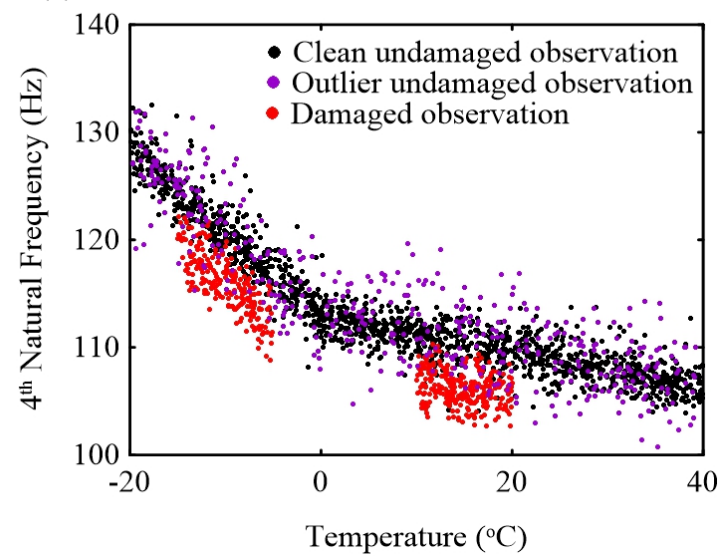

Figure 13: Plot of temperature versus (a) first natural frequency, (b) second natural frequency, (c) thrid natural frequency and (d) fourth natural frequency of clean undamaged observations, outlier undamaged observations and damaged observations (only for temperatures of 10 ${ }^{\circ} \mathrm{C}$ to $20{ }^{\circ} \mathrm{C}$ and $-5{ }^{\circ} \mathrm{C}$ to $-15{ }^{\circ} \mathrm{C}$ ) of the beam structure model.

\subsection{Z24 Bridge}

The Z24 Bridge (Fig. 15), a post-tensioned concrete girder bridge, was located in Switzerland connecting Koppigen and Utzenstorf and overpassing the A1 highway. It was a three spans bridge with a main span of $30 \mathrm{~m}$ and ${ }^{875}$ two side spans of $14 \mathrm{~m}$ each. It was monitored for almost a year to collect different environmental parameters well as acceleration measurements. The acceleration measurements were recorded for almost every hour and an automatic system identification system was in place to de-880 rive the modal parameters of the bridge. The bridge was gradually damaged near the end of the monitoring period itoring. The damaged cases that the bridge was subjected with are presented in Table 2 and a detailed description of ${ }^{88}$ the cases can be found in Kramer et al. 42 .

The first four natural frequencies of the bridge are used as damage sensitive features in this paper. A bilinear relationship between the four natural frequencies of the undamaged cases and ambient temperature conditions can ${ }^{890}$ be found as shown in Fig. 16. Peeters and De Roeck 5. suggested that the bilinear relationship was attributed to tributed to the increase in stiffness of the structure, while at warmer temperatures, it had less influence.

The damaged cases are also included in Fig. 16. It can be seen that for the first, third and fourth natural frequencies, the damaged cases lie mostly in the space containing the outlier observations (undamaged observations that are far away from the majority of the undamaged measurements). Therefore, as mentioned previously, it is important to clean that space so that smaller damaged cases can be detected using damage detection methods. For the second natural frequency, the undamaged and damaged cases are separated from each other.

The first 4000 undamaged observations are used as the undamaged database used to create the baseline, while the rest of the observations are used for testing. It should be noted that the natural frequencies of some observations were not extracted, hence, these observations cannot be analysed.

PCA is first applied to the undamaged database used to create the baseline. Fig. 17 gives the plots of the principal component scores arranged against observation number. Two different regions can be seen in the first principal component plot. This represents the behaviour of 
(a)

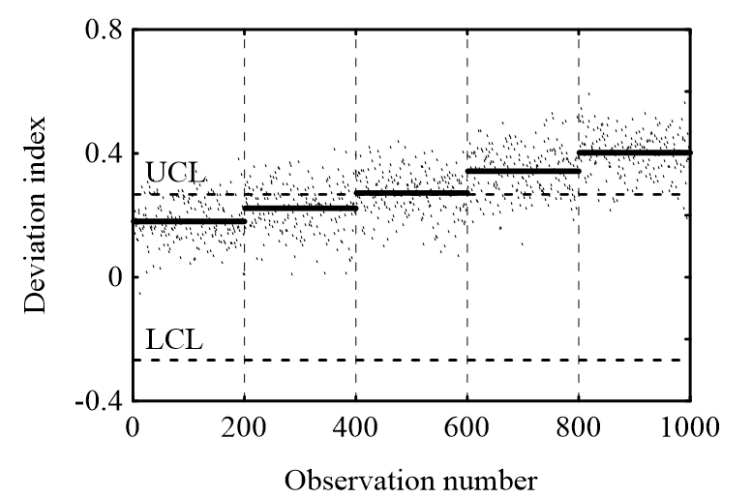

(b)

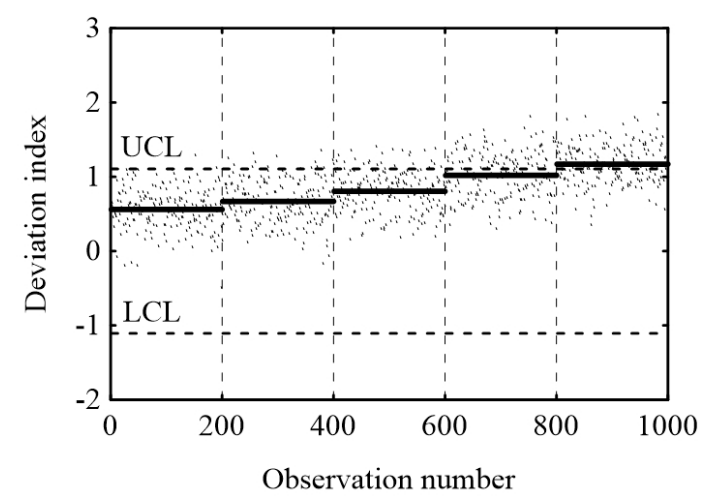

(c)

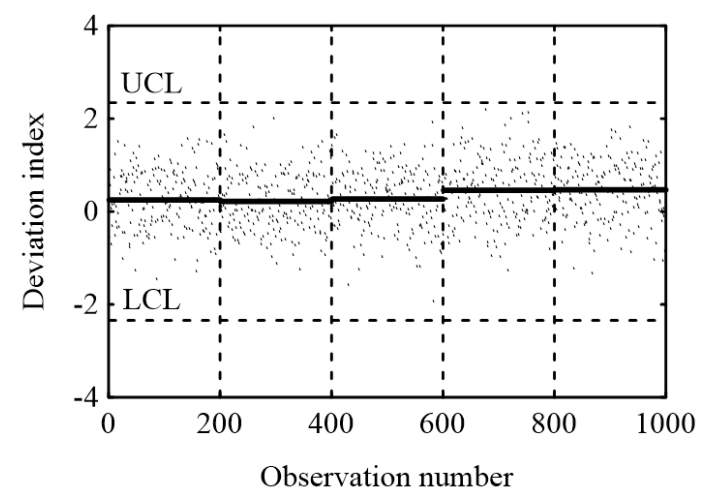

(d)

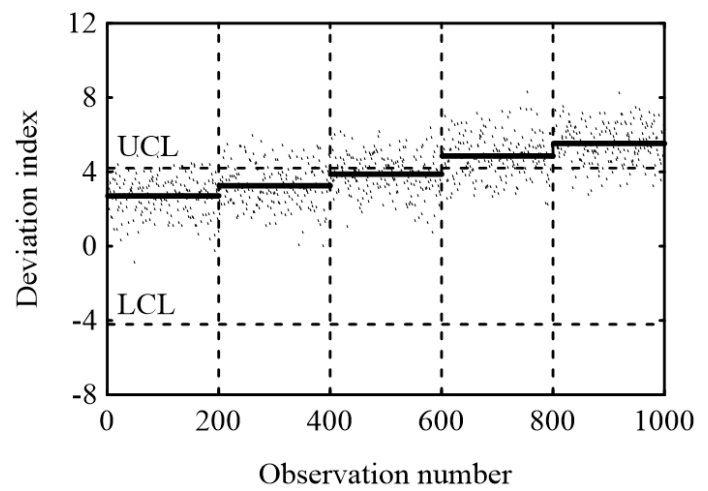

Original database
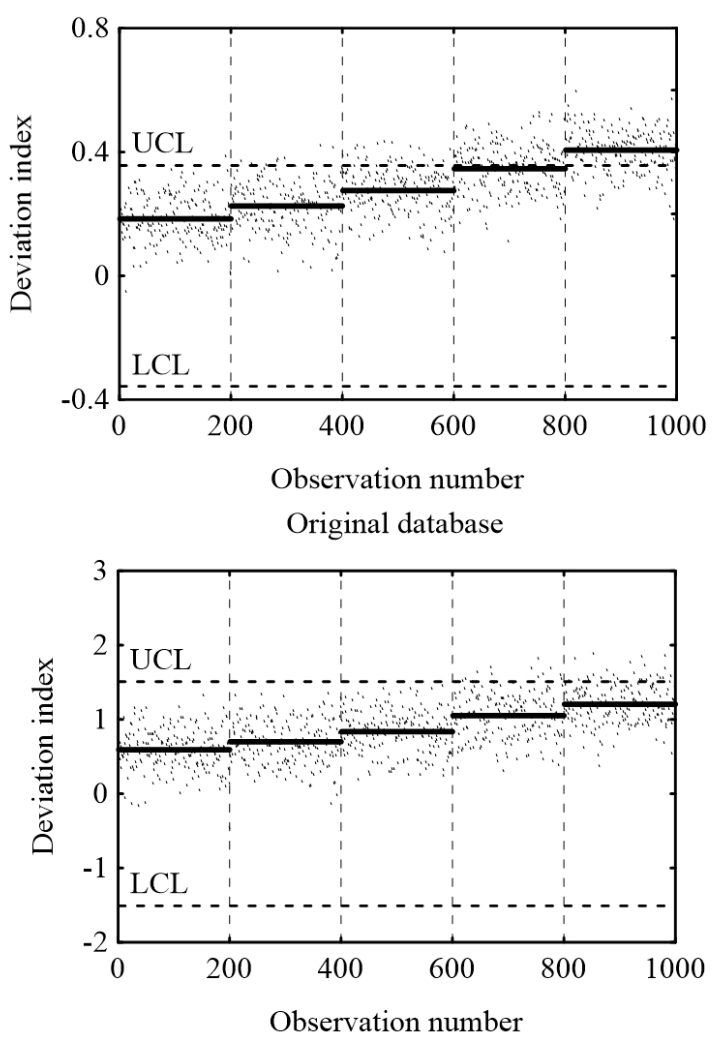

Original database

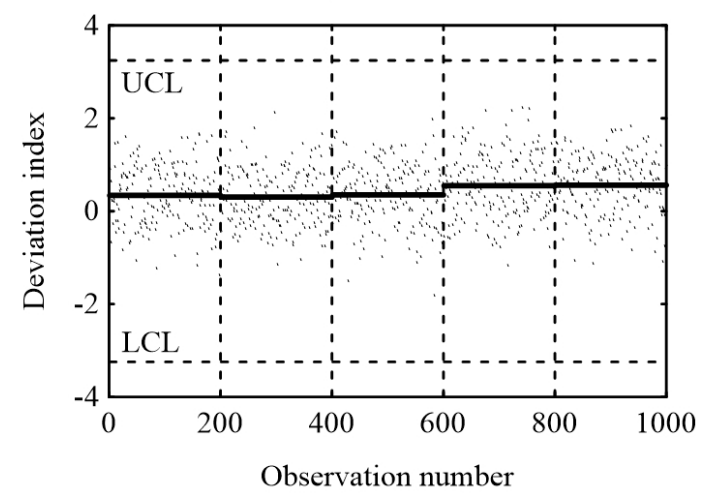

Original database

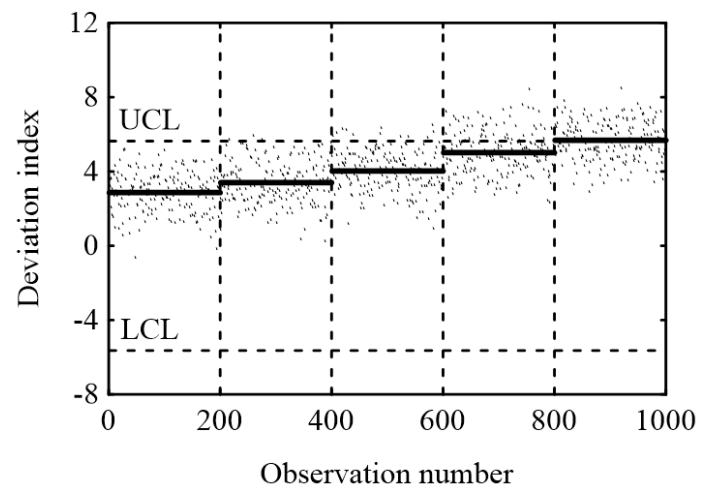

Figure 14: Results of outlier analysis for (a) first natural frequency, (b) second natural frequency, (c) third natural frequency and (d) fourth natural frequency using the clean database (left-hand side) and the original database (right-hand side) for the beam structure model. *0-200 observations: $25 \%$ damage, 201:400 observations: $30 \%$ damage, 401:600 observations: $35 \%$ damage, 601:800 observations: $40 \%$ damage and 801:1000 observations: $45 \%$ damage. 


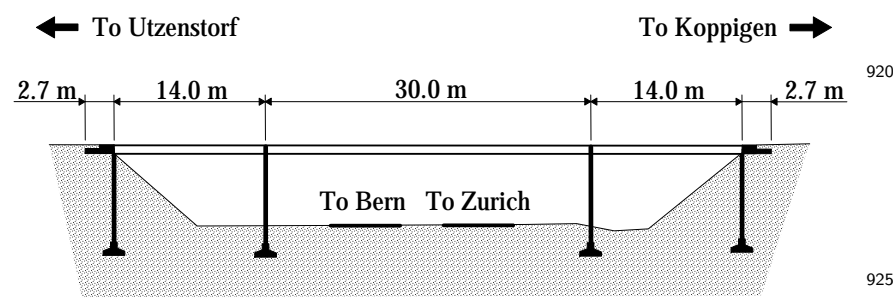

Figure 15: Z24 Bridge (Adapted from Peeters and De Roeck[5]).

Table 2: Description of the progressive damage cases applied to the $Z_{24}{ }_{930}$ Bridge (Adapted from Reynders et al. [40]).

\begin{tabular}{ll}
\hline Test $\mathrm{N}^{-}$ & Case description \\
\hline 1 & Reference state \\
2 & System installation for pier settlement \\
3 & $20 \mathrm{~mm}$ settlement of pier \\
4 & $40 \mathrm{~mm}$ settlement of pier \\
5 & $80 \mathrm{~mm}$ settlement of pier \\
6 & $95 \mathrm{~mm}$ settlement of pier \\
7 & Foundation tilt \\
8 & New reference state \\
9 & $12 \mathrm{~m}^{2}$ chipping of concrete \\
10 & $24 \mathrm{~m}^{2}$ chipping of concrete \\
11 & Landslide \\
12 & Concrete hinges failure \\
13 & 2 anchor heads failure \\
14 & 4 anchor heads failure \\
15 & Rupture of 2 tendons out of 16 \\
16 & Rupture of 4 tendons out of 16 \\
17 & Rupture of 6 tendons out of 16 \\
\hline
\end{tabular}
damage alert. This will prevent classifying undamaged measurements with high noise level as damage.

\section{Conclusion}

A wide range of damage detection methods have been proposed in the literature. However, most of these methods create the baseline of the undamaged structure with outlier measurements present in the training database. Even though damage can be identified, these outlier measurements prevent the methods from alerting small damage. Therefore, an approach is proposed in this paper to identify these outliers to allow a clean baseline to be constructed. The proposed approach makes use of Principal Component Analysis and Median Absolute Deviation to highlight the outlier measurements. As per knowledge of the authors, it is the first time that Median Absolute Deviation is used in the context of damage detection of civil engineering structures under changing environmental and operational conditions. A beam structure model and the Z24 Bridge are analysed using the proposed approach and the results demonstrate that damage can be detected at an earlier stage. The results obtained also highlight the importance of identifying outliers before the application of damage detection methods, because a defective model of the undamaged structure can be created if these outliers are not taken into account.

\section{Acknowledgment}

The authors would like to acknowledge Prof. Guido De Roeck and Prof. Edwin Reynders for providing the data of the Z24 Bridge. This work was financially supported by the National Science Foundation of China (51708306, 51605233), Ningbo Natural Science Program (2018A610352) and Faculty Inspiration Grant.

\section{References}

[1] H. Sohn, Effects of environmental and operational variability on structural health monitoring, Philos Trans Royal Soc A 365 (1851) (2007) 539-560. 
(a)

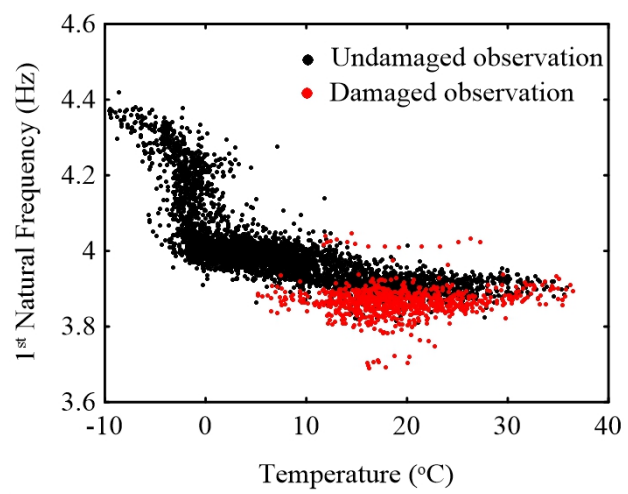

(c)

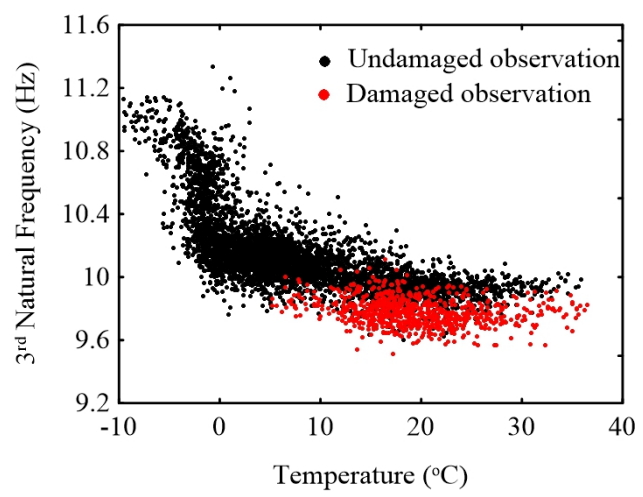

(b)

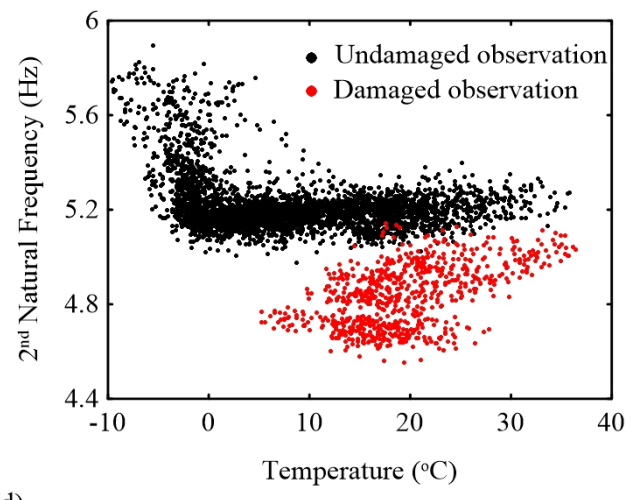

(d)

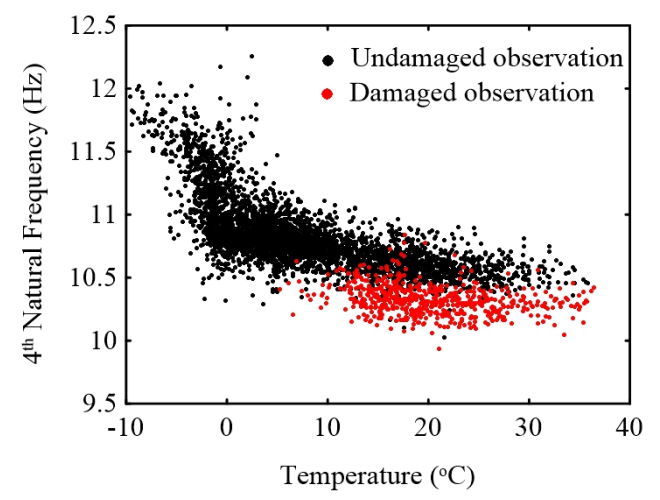

Figure 16: Plot of temperature versus (a) first natural frequency, (b) second natural frequency, (c) third natural frequency and (d) fourth natural frequency of the Z24 Bridge.

(a)

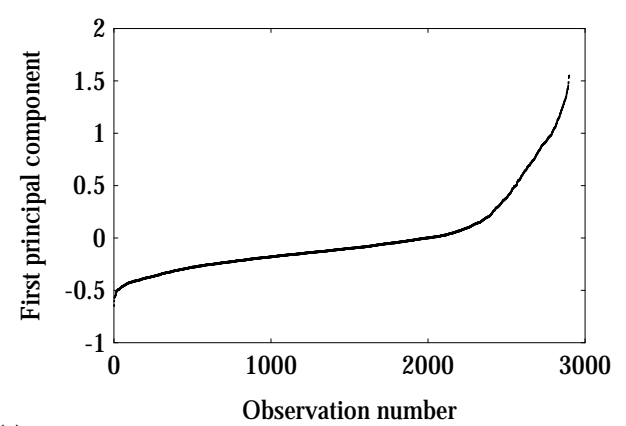

(c)

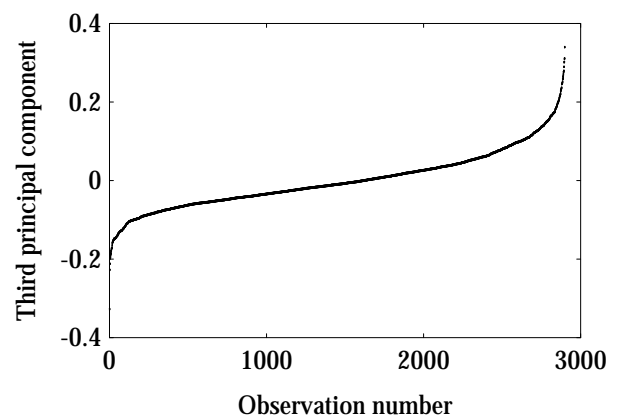

(b)

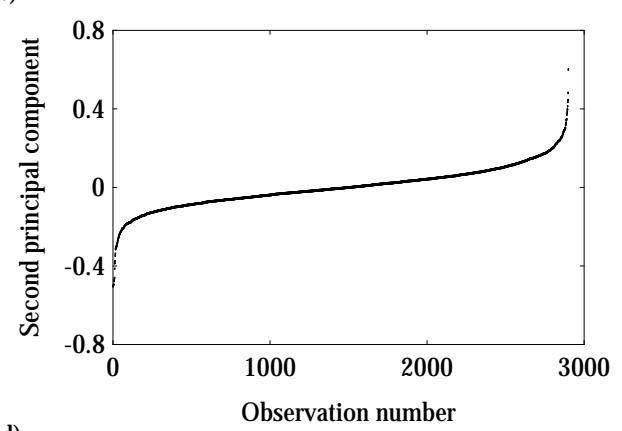

(d)

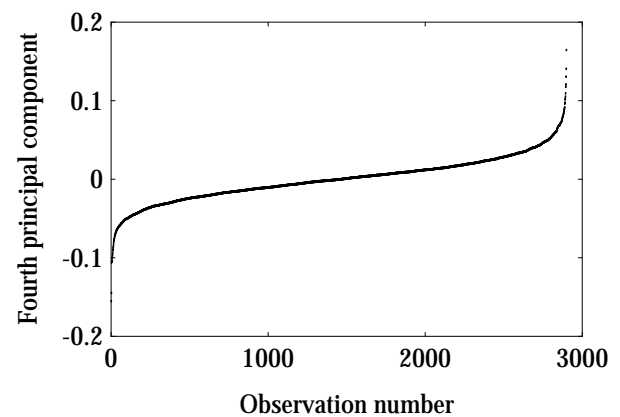

Figure 17: Plot of (a) first principal component, (b) second principal component, (c) third principal component and (d) fourth principal component arranged against observation number for the database of the Z24 Bridge. 
Table 3: Successful rate of the damage detection method applied to the Z24 Bridge.

\begin{tabular}{lllll}
\hline & \multicolumn{2}{c}{ Damaged cases } & \multicolumn{2}{c}{ Undamaged cases } \\
\hline Natural frequency & Clean database (\%) & Original database (\%) & Clean database (\%) & Original database (\%) \\
\hline 1 & 36.7 & 29 & 97.6 & 98.8 \\
2 & 98.9 & 98.7 & 94.9 & 97.5 \\
3 & 30.7 & 13.3 & 98.2 & 99.4 \\
4 & 49.7 & 30.7 & 98.2 & 99.5 \\
\hline
\end{tabular}

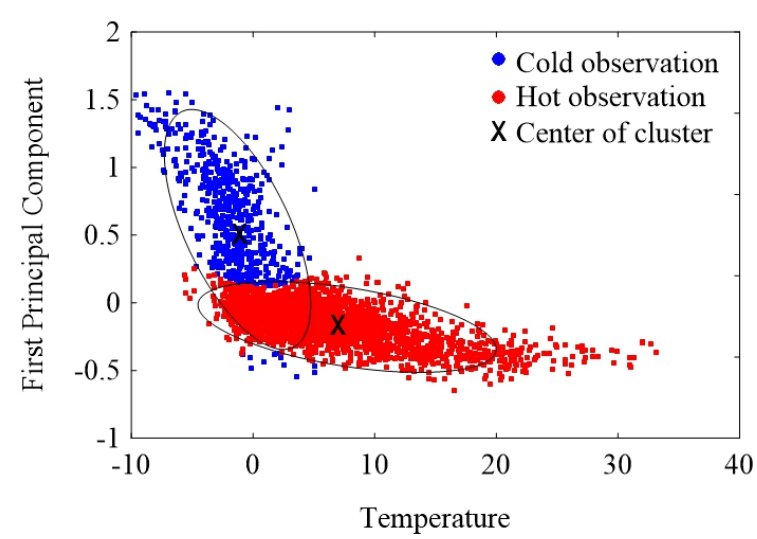

Figure 18: Cluster of first principal component with temperature of the Z24 Bridge using GMM.

[2] W. Soo Lon Wah, Y.-T. Chen, A new approach toward dam1030 age localization and quantification of structures under changing temperature condition, J Low Freq Noise V A.

[3] C. Fritzen, G. Mengelkamp, A. Guemes, Elimination of temperature effects on damage detection within a smart structure concept, in: Proceedings of the 4th International Workshop on 035 Structural Health Monitoring, Stanford, CA, 15-17 September 2003, pp. 1530-1538.

[4] N. Dervilis, K. Worden, E. Cross, On robust regression analysis as a means of exploring environmental and operational conditions for shm data, J Sound Vib 347 (2015) 279-296.

[5] B. Peeters, G. De Roeck, One-year monitoring of the z24-bridge: environmental effects versus damage events, Earthq Eng Struct Dyn 30 (2) (2001) 149-171.

[6] K. Worden, H. Sohn, C. Farrar, Novelty detection in a changing environment: regression and interpolation approaches, J Sound045 Vib 258 (4) (2002) 741-761.

[7] B. Peeters, J. Maeck, G. De Roeck, Dynamic monitoring of the z24-bridge: separating temperature effects from damage, in: Proceedings of the European COST F3 Conference on System Identification and Structural Health Monitoring, Madrid, Spainı̧050 June 2000, pp. 377-386.

[8] Y.-Q. Ni, K. Fan, G. Zheng, T. H. Chan, J. M. Ko, Automatic modal identification of cable-supported bridges instrumented with a long-term monitoring system, in: Smart Structures and Materials 2003: Smart Systems and Nondestructive Evaluation 055 for Civil Infrastructures, San Diego, CA, March 2003, pp. 329339 .

[9] C. Gentile, M. Guidobaldi, A. Saisi, One-year dynamic monitoring of a historic tower: damage detection under changing environment, Meccanica 51 (11) (2016) 2873-2889.

[10] E. J. Cross, K. Worden, Q. Chen, Cointegration: a novel approach for the removal of environmental trends in structural health monitoring data, P Roy Soc Lond A Mat 467 (2133) (2011) 2712-2732.

[11] G. Comanducci, F. Ubertini, A. L. Materazzi, Structural healtho6s monitoring of suspension bridges with features affected by changing wind speed, J Wind Eng Ind Aerod 141 (2015) 12-
26.

12] W. Soo Lon Wah, Y.-T. Chen, G. W. Roberts, A. Elamin, Separating damage from environmental effects affecting civil structures for near real-time damage detection, Struct Health Monit.

[13] W. Soo Lon Wah, Y.-T. Chen, G. W. Roberts, A. Elamin, Damage detection of structures subject to nonlinear effects of changing environmental conditions, in: The 6th Asia-Pacific Workshop on Structural Health Monitoring, Vol. 188, Elsevier, 2017, pp. 248-255.

[14] W. Soo Lon Wah, J. S. Owen, Y.-T. Chen, A. Elamin, G. W. Roberts, Damage detection of a cable-stayed bridge subjected to changing environmental and operational conditions, in: The 7th World Conference on Structural Control and Monitoring, Qingdao, China, 22-25 July 2018.

[15] F. Magalhães, A. Cunha, E. Caetano, Vibration based structural health monitoring of an arch bridge: from automated oma to damage detection, Mech Syst Signal Process 28 (2012) 212228.

[16] A. Cabboi, C. Gentile, A. Saisi, From continuous vibration monitoring to fem-based damage assessment: application on a stonemasonry tower, Constr Build Mater 156 (2017) 252-265.

[17] F. Ubertini, N. Cavalagli, A. Kita, G. Comanducci, Assessment of a monumental masonry bell-tower after 2016 central italy seismic sequence by long-term shm, B Earthq Eng 16 (2) (2018) $775-801$.

[18] D. M. Hawkins, Identification of outliers, Vol. 11, Springer, 1980.

[19] F. Pedregosa, G. Varoquaux, A. Gramfort, V. Michel, B. Thirion, O. Grisel, M. Blondel, P. Prettenhofer, R. Weiss, V. Dubourg, J. Vanderplas, A. Passos, D. Cournapeau, M. Brucher, M. Perrot, E. Duchesnay, Scikit-learn: Machine learning in Python user guide.

[20] R. Fuentes, On bayesian networks for structural health and condition monitoring, Ph.D. thesis, University of Sheffield (2017).

[21] P. Moser, B. Moaveni, Environmental effects on the identified natural frequencies of the dowling hall footbridge, Mech Syst Signal Process 25 (7) (2011) 2336-2357.

[22] C. Jin, J. Li, S. Jang, X. Sun, R. Christenson, Structural damage detection for in-service highway bridge under operational and environmental variability, in: Sensors and Smart Structures Technologies for Civil, Mechanical, and Aerospace Systems 2015, Vol. 9435, International Society for Optics and Photonics, 2015.

[23] P. Salcher, H. Pradlwarter, C. Adam, Reliability assessment of railway bridges subjected to high-speed trains considering the effects of seasonal temperature changes, Eng Struct 126 (2016) $712-724$.

[24] K. Worden, G. Manson, N. R. Fieller, Damage detection using outlier analysis, J Sound Vib 229 (3) (2000) 647-667.

[25] E. Cross, K. Koo, J. Brownjohn, K. Worden, Long-term monitoring and data analysis of the tamar bridge, Mech Syst Signal Process 35 (1-2) (2013) 16-34.

[26] C. R. Farrar, S. W. Doebling, P. J. Cornwell, E. G. Straser, Variability of modal parameters measured on the alamosa canyon bridge, Tech. rep., Los Alamos National Lab., NM (United States) (1996).

[27] I. T. Jolliffe, Springer series in statistics, Principal component analysis 29 .

[28] S. Alampalli, Influence of in-service environment on modal pa- 

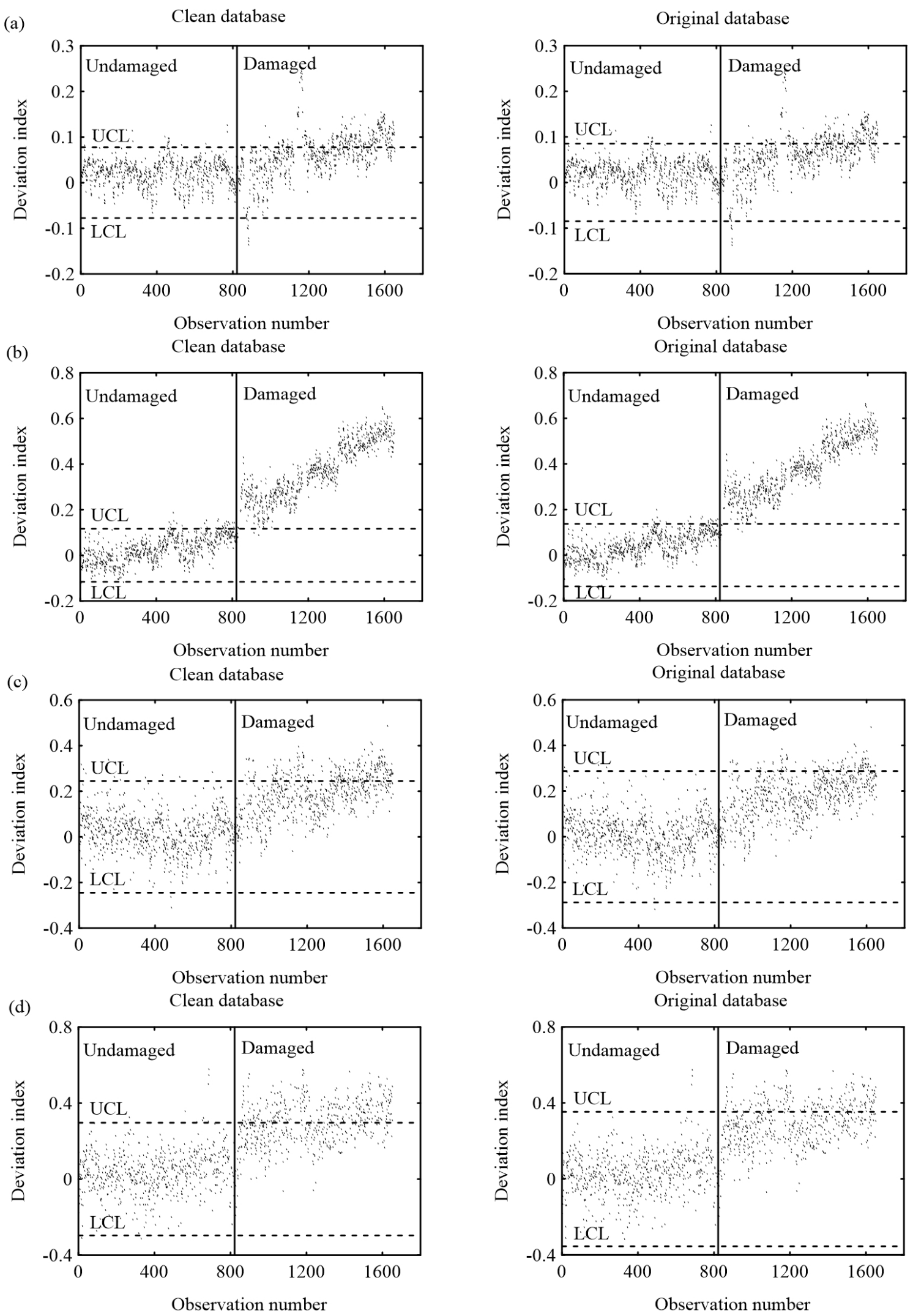

Figure 19: Results of outlier analysis for (a) first natural frequency, (b) second natural frequency, (c) third natural frequency and (d) fourth natural frequency using the clean database (left-hand side) and the original database (right-hand side) for the Z24 Bridge. 
rameters, in: Proceedings of the 16th International Modal Analysis Conference, Santa Barbara, CA, 2-5 February 1998, pp. 111-116.

[29] J. M. Ko, K. K. Chak, J. Y. Wang, Y.-Q. Ni, T. H. Chan, Formulation of an uncertainty model relating modal parameters and environmental factors by using long-term monitoring data, in: Proceedings of Smart Structures and Materials 2003: Smart Systems and Nondestructive Evaluation for Civil Infrastructures, San Diego, CA, 2-6 March 2003, pp. 298-307.

[30] X. He, J. P. Conte, M. Fraser, A. Elgamal, Long-term monitoring of a highway bridge, in: Proceedings of the 3rd International Operational Modal Analysis Conference, Portonovo, Italy, 4-6 May 2009, pp. 29-36.

[31] S. Desjardins, N. Londono, D. Lau, H. Khoo, Real-time data processing, analysis and visualization for structural monitoring of the confederation bridge, Adv Struct Eng 9 (1) (2006) 141157.

[32] C. Leys, C. Ley, O. Klein, P. Bernard, L. Licata, Detecting outliers: Do not use standard deviation around the mean, use absolute deviation around the median, J Exp Soc Psychol 49 (4) (2013) 764-766.

[33] D. L. Donoho, P. J. Huber, The notion of breakdown point, A festschrift for Erich L. Lehmann 157184.

[34] P. J. Rousseeuw, C. Croux, Alternatives to the median absolute deviation, J Am Stat assoc 88 (424) (1993) 1273-1283.

[35] G. Kerschen, J.-C. Golinval, Non-linear generalization of principal component analysis: from a global to a local approach, J Sound Vib 254 (5) (2002) 867-876.

[36] A.-M. Yan, G. Kerschen, P. De Boe, J.-C. Golinval, Structural damage diagnosis under varying environmental conditionspart ii: local pca for non-linear cases, Mech Syst Signal Process 19 (4) (2005) 865-880.

[37] J. Kullaa, Structural health monitoring under nonlinear environmental or operational influences, Shock and Vibration 2014.

[38] C. M. Bishop, Pattern Recognition and Machine Learning (Information Science and Statistics), Springer-Verlag, 2006.

[39] A. Cunha, E. Caetano, P. Ribeiro, Statistical analysis of the damage detection performance under environmental or operational influences.

[40] E. Reynders, G. Wursten, G. De Roeck, Output-only structural health monitoring in changing environmental conditions by means of nonlinear system identification, Struct Health Monit 13 (1) (2014) 82-93.

[41] J. Kullaa, Distinguishing between sensor fault, structural damage, and environmental or operational effects in structural health monitoring, Mech Syst Signal Process 25 (8) (2011) 2976 2989.

[42] C. Krämer, C. De Smet, G. De Roeck, Z24 bridge damage detection tests, in: Proceedings of the 17th International Modal Analysis Conference, Kissimmee, FL, 8-11 February 1999, pp. 1023-1029. 